\title{
Emission line galaxies and active galactic nuclei in WINGS clusters ${ }^{\star}$
}

\author{
P. Marziani ${ }^{1}$, M. D’Onofrio ${ }^{1,2}$, D. Bettoni ${ }^{1}$, B. M. Poggianti ${ }^{1}$, A. Moretti ${ }^{1}$, \\ G. Fasano ${ }^{1}$, J. Fritz ${ }^{3}$, A. Cava ${ }^{4}$, J. Varela ${ }^{5}$, and A. Omizzolo ${ }^{1,6}$ \\ 1 INAF, Osservatorio Astronomico di Padova, vicolo dell' Osservatorio 5, 35122 Padova, Italy \\ e-mail: paola.marziani@oapd.inaf.it \\ 2 Dipartimento di Fisica \& Astronomia “Galileo Galilei”, Università di Padova, vicolo dell' Osservatorio 3, \\ 35122 Padova, Italy \\ 3 Centro de Radioastronomía y Astrofísica, CRyA, UNAM, Michoacán, Mexico \\ ${ }^{4}$ Observatoire de Genève, Université de Genève, 51 Ch. des Maillettes, 1290 Versoix, Switzerland \\ 5 Centro de Estudios de Física del Cosmos de Aragón (CEFCA), Plaza San Juan 1, planta 2, 44001 Teruel, Spain \\ ${ }^{6}$ Specola Vaticana, 00120 Vatican City State, Italy
}

Received 16 May 2016 / Accepted 23 August 2016

\begin{abstract}
We present the analysis of the emission line galaxies members of 46 low-redshift $(0.04<z<0.07)$ clusters observed by WINGS (WIde-field Nearby Galaxy cluster Survey). Emission line galaxies were identified following criteria that are meant to minimize biases against non-star-forming galaxies and classified employing diagnostic diagrams. We examined the emission line properties and frequencies of star-forming galaxies, transition objects, and active galactic nuclei (AGNs: LINERs and Seyferts), unclassified galaxies with emission lines, and quiescent galaxies with no detectable line emission. A deficit of emission line galaxies in the cluster environment is indicated by both a lower frequency, and a systematically lower Balmer emission line equivalent width and luminosity with respect to control samples; this implies a lower amount of ionized gas per unit mass and a lower star formation rate if the source is classified as HII region. A sizable population of transition objects and of low-luminosity LINERs $(\approx 10-20 \%$ of all emission line galaxies) are detected among WINGS cluster galaxies. These sources are a factor of $\approx 1.5$ more frequent, or at least as frequent, as in control samples with respect to HII sources. Transition objects and LINERs in clusters are most affected in terms of line equivalent width by the environment and appear predominantly consistent with so-called retired galaxies. Shock heating can be a possible gas excitation mechanism that is able to account for observed line ratios. Specific to the cluster environment, we suggest interaction between atomic and molecular gas and the intracluster medium as a possible physical cause of line-emitting shocks.
\end{abstract}

Key words. catalogs - galaxies: clusters: general - galaxies: clusters: intracluster medium - galaxies: star formation galaxies: statistics - galaxies: evolution

\section{Introduction}

Many galaxy properties depend on their environment (see, e.g., Sulentic 1976; Larson \& Tinsley 1978, for pionieering work, and, e.g., Lee et al. 2003; Poggianti et al. 2006; Bernardi et al. 2006; Blanton \& Moustakas 2009; Scoville et al. 2013; Bitsakis et al. 2016, for more modern perspectives). At one extreme we find the most isolated galaxies whose morphology may be a fossil of early times of galaxy formation (Verdes-Montenegro et al. 2005). At the other extreme we find galaxies living in the very dense environment of clusters, where morphology and gas content are affected by frequent intergalaxy gravitational interactions such as minor and major merging, various forms of tidal harassments involving $\mathrm{HI}$ disk truncation, and tidal stripping of gas and stars (e.g., Dale et al. 2001; Park \& Hwang 2009). If we were only considering gravitational effects, a naïve expectation would be to find a large percentage of luminous emission line galaxies (ELGs) hosting an active nucleus among cluster galaxies. Strong gravitational interactions

\footnotetext{
* The data whose description is provided in Table B.1, and emission line catalog of the WINGS database are only available at the CDS via anonymous ftp to cdsarc.u-strasbg. fr (130.79.128.5) or via http://cdsarc.u-strasbg.fr/viz-bin/qcat?J/A+A/599/A83
}

are known to provide a trigger for nuclear activity over a wide range of luminosity, if one of the galaxies involved in the interaction is sufficiently gas rich (Hwang et al. 2012; Sabater et al. 2013). It has been recognized for over 30 years that this is not the case of cluster environments, most likely because of the interaction of the galaxy atomic and molecular gas with the hot intra-cluster medium (ICM) whose pressure is higher in the inner cluster core $\left(\$ 0.5 r_{\mathrm{vir}}\right)$. The dynamical action of the ICM on a galaxy interstellar gas may lead to its stripping by ram pressure. Ram stripping is expected to influence the evolution of galaxies in clusters to the point of even transforming a spiral galaxy into an anemic S0 galaxy (e.g., Spitzer \& Baade 1951; Gunn \& Gott 1972; Giovanelli \& Haynes 1985; Boselli \& Gavazzi 2006; D'Onofrio et al. 2015). Systematic effects of ram stripping have been traced by a deficit of HI gas, spectacularly illustrated for the Virgo cluster (Cayatte et al. 1990), and by a deficit of ELGs in cluster environments (e.g., Balick \& Heckman 1982; Hwang et al. 2012; Pimbblet et al. 2013).

Of particular interest in this context is the problem of the AGN fraction and location in galaxy clusters (e.g., Miller et al. 2003; Kauffmann et al. 2004; Choi et al. 2009; Martini et al. 2006, 2009; Haggard et al. 2010; von der Linden et al. 2010; Hwang et al. 2012; Pimbblet et al. 2013). Previous works often 
conclude with contrasting results concerning the role of the environment even when the extended spectral database provided by the SDSS is used. It is possible that the origin of these discrepancies is linked to the employed data samples as well as to analysis techniques adopted for the emission line spectra. It is also possible that two major open issues concerning ELGs and their properties plague any conclusion about the AGN fraction and the role of the cluster environment: first, the classification of galaxies as AGNs by means of diagnostic diagrams (DDs) might vary with the DD used and strongly depends on the signal-to-noise ratio $(\mathrm{S} / \mathrm{N})$ of the available spectra; second, the understanding of the physical nature of faint ELGs as possible AGNs is far from definitive.

Faint emission lines are buried in the absorption spectrum of the host galaxy, as spectra are often obtained with a fiber covering a significant spatial extent of the host galaxies. Diagnostic diagrams are customarily used for the classification of ELGs. They however require the accurate measurement of three or four emission lines. Currently, the DDs proposed by Veilleux \& Osterbrock (1987) are most frequently used; these have the considerable advantage of employing line pairs that are proximate in wavelength. The downside is that two of three DD-related lines are fairly weak, and they require a measure of the $\mathrm{H} \alpha$ and $\mathrm{H} \beta$ emission lines that are most affected by the absorption spectrum of the host galaxy.

The second issue is related to the physical nature of ELGs. Once a source is placed in a DD, the customary subdivision involves a net separation of sources in three classes: HII, LINERs, and Seyfert sources, the latter two often collectively referred to as AGNs. In the DD involving [NII] $\lambda 6583 / \mathrm{H} \alpha$ vs. $[\mathrm{OIII}] \lambda 5007 / \mathrm{H} \beta$ a finer subdivision has been introduced. A line based on the theoretical maximum line ratios, which are possible from pure stellar photoionization, distinguishes HII regions from other ELGs (Kewley et al. 2001). Sources above this line (i.e., with larger [OIII] $\lambda 5007 / \mathrm{H} \beta$ ratios) are likely dominated by emission associated with non-stellar sources of ionization as provided by active nuclei. The Kewley et al. (2001) dividing line comes from a photoionization analysis that includes extreme conditions for line emission from gas ionized by stellar sources. A further subdivision (Kauffmann et al. 2003) distinguishes between pure star-forming galaxies and Seyferts and composite objects or transition objects (TOs), i.e., sources located in between the region of LINERs and the HII region in the [OIII] $\lambda 5007 / \mathrm{H} \beta$ vs. [NII] $\lambda 6583 / \mathrm{H} \alpha$ DD. This latter line is purely empirical. While classification is safe and not questioned for most HII regions, the physical interpretation of the DDs involves two controversial aspects. First, Seyferts, with high ionization spectra, and LINERs, with low ionization spectra by definition, are included in the broader class of AGNs; and second, TOs show properties that may not be fully consistent with photoionization by hot stars but still lie within the region of theoretically admissible HII region.

Most galaxies in the field show emission lines, if the equivalent width is as low as $0.25 \AA$. Their line luminosity is correspondingly low $\left(\sim 10^{38} \mathrm{erg} \mathrm{s}^{-1}\right.$, Ho et al. 1995) with an open-ended lower limit (Stasińska et al. 2008). In keeping with the AGN hypothesis for low-luminosity (LL) ELGs $(L(\mathrm{H} \alpha) \lesssim$ $10^{40} \mathrm{erg} \mathrm{s}^{-1}$ ) that fall outside of the HII + TO region, these lowluminosity sources may be associated with massive black holes that are accreting at exceedingly low rates $\left(\dot{m} \sim 10^{-3}-10^{-5}\right.$; Ho 2005), which are well below the rates of luminous Seyfert 1 galaxies $\left(\dot{m} \gtrsim 10^{-2}\right)$. Such sources represent a sizable fraction of all galaxies with detected emission lines, whereas Seyfert 1 and 2 represent $1 \%$ and $3 \%$, respectively, of the low-z SDSS galaxies (Hao et al. 2005). If they are really AGNs, the classical fueling problem has manifold solutions because of the low accretion rates involved. Accretion material may even be due to mass loss by evolved stars in the nuclear regions of a galaxy (Padovani \& Matteucci 1993; Heckman \& Best 2014).

This interpretation of LINERs, which usually have low luminosity and are a large percentage of LL ELGs, as AGNs assumes that line emission is due to gas that is photoionized by a continuum harder than that of hot, massive stars. The excess X-ray emission of AGNs leads to an excess of low ionization lines (in the DD case, [OI] $\lambda 6300$ and [SII] $\lambda \lambda 6716,6731$ ) with respect to the Balmer lines and high ionization species (in the DD case, [OIII] $\lambda \lambda 4959$, 5007; Veilleux \& Osterbrock 1987; Kewley et al. 2001). Originally defined from the conditions $[\mathrm{OII}] \lambda 3727 /[\mathrm{OIII}] \lambda 5007 \geq 1$ and $[\mathrm{OI}] \lambda 6300 /[\mathrm{OIII}] \lambda 5007 \leq \frac{1}{3}$ (Heckman 1980), LINERs, are certainly a heterogeneous class of sources. The AGN interpretation is not the only possible explanation, especially in the LL domain. Sources classified as low-luminosity AGNs may not only be true LL AGNs (Coziol et al. 2014), but also galaxies whose emission line gas is ionized by post-asymptotic branch stars (Cid Fernandes et al. 2010) or even shock heated (e.g., Newman et al. 2014, and references therein). The dominant interpretation of LINERs is that of LLAGNs due to the detection of a nuclear compact hard-X source in most of these galaxies (González-Martín et al. 2006; Márquez et al. 2007; Anderson et al. 2007). Some LINERs can be Compton-thick, and they may be missed in soft-X ray observations (0.2-2 keV, González-Martín et al. 2015). However, the LINER optical emission line spectrum can be produced by photoionization (Ferland \& Netzer 1983; Halpern \& Steiner 1983) and, as demonstrated since the early 1980s, this spectrum can be equally well produced by shock heating (Contini \& Aldrovandi 1983; Viegas-Aldrovandi \& Gruenwald 1990). Shocks and postAGB photoionization are not exclusive to the AGN scenario and may help explain why the prevalence of these sources is high in elliptical and early-type spirals (Kauffmann 2009).

If we now turn to clusters, surveys like the Sloan Digital Sky Survey (SDSS) and WIde field Nearby Galaxy Cluster Survey (WINGS; Fasano et al. 2006) opened up the possibility of using large samples. However, observations of individual clusters cover at best $\sim 100$ cluster members and, more frequently, a few tens. These numbers are insufficient to satisfactorily sample the relatively rare AGN phenomenon whose prevalence is a strong function of luminosity and AGN class: LINER-type activity may be detected in one-third of early-type galaxies at $L \sim 2 \times 10^{39} \mathrm{erg} \mathrm{s}^{-1}$ (Ho et al. 1997; Carrillo et al. 1999), but luminous Seyfert 1s may not exceed $1 \%$ of galaxies in the local Universe (Huchra \& Burg 1992; Ho et al. 1995). In addition, the ionized gas content of cluster galaxies is expected to be lower than in the field. This makes the study of emission lines in cluster galaxy spectra even more challenging, requiring very high $\mathrm{S} / \mathrm{N}$ at intermediate resolution to ensure that faint emission components are detected.

This paper deals the problems concerning the AGN fraction in the clusters mentioned above, taking advantage of the WINGS spectral database (Fasano et al. 2006; Varela et al. 2009; Cava et al. 2009; Moretti et al. 2014). The spectra of WINGS are adequate in terms of both $\mathrm{S} / \mathrm{N}$ and dispersion to sample the faint non-HII population (i.e., Seyfert, LINER, and TO) at typical equivalent widths $\sim 1 \AA$, but again the relative rarity of detectable nuclear activity requires that survey clusters are stacked together for a prevalence study of AGN classes (Sect. 2.2).

The sample of cluster galaxies for the present investigation is described in Sect. 2, along with a summary of the main instrumental properties that characterize the spectroscopic data. 
Measurements are discussed in Sect. 3, with data analysis including error estimates and censored data in Appendix A. Results of emission line measurements are reported in Sect. 4 and are statistically analyzed in Sect. 4.3 and later subsections. The discussion of emission line measurements (Sect. 5) is mainly focused on a comparison with previous works involving ELGs statistics in clusters (Sect. 5.3) and in different environments (Sect. 5.4), and especially on the possibility of interpreting low-luminosity, nonHII ELGs as predominantly shock-heated sources (Sect. 5.2). We assume cosmological parameters $H_{0}=70 \mathrm{~km} \mathrm{~s}^{-1} \mathrm{Mpc}^{-1}$, $\Omega_{\mathrm{M}}=0.3, \Omega_{\Lambda}=0.7$.

\section{Sample definition}

\subsection{The WINGS cluster sample}

The survey WINGS (Fasano et al. 2006) ${ }^{1}$ is an imaging and spectroscopic study of the brightest X-ray clusters at redshift $0.04<z<0.07$ selected from the ROSAT all sky survey. The basic properties of the 46 clusters considered in this study are reported in Table 1. Table 1 lists the cluster name, redshift, velocity dispersion and associated uncertainty in $\mathrm{km} \mathrm{s}^{-1}$, logarithm of soft X-ray luminosity (0.5-2.0 keV) as in Fasano et al. (2006), and the virial radius $R_{200}$ in Mpc. The following two columns report, for each cluster, the numbers of sources observed that are spectroscopically confirmed cluster members $\left(N_{1}\right)$ and those that are non-members $\left(N_{0}\right)$ following the criterion of Cava et al. (2009). The last column label indicates if a cluster was observed from the northern or southern Hemisphere, with somewhat different instrumental setups (see Sect. 2.2).

The low-redshift sample includes clusters with velocity dispersion in the range $500 \mathrm{~km} \mathrm{~s}^{-1} \lesssim \sigma \lesssim 1100 \mathrm{~km} \mathrm{~s}^{-1}$, and $43.5 \lesssim \log L_{\mathrm{X}} \lesssim 45$ [erg s${ }^{-1}$ ]. These properties place the WINGS clusters toward the high end of the cluster mass and X-ray luminosity function in the local Universe (Bahcall \& Cen 1992; Biviano et al. 1993; Girardi et al. 1998; Böhringer et al. 2014; Reiprich \& Böhringer 2002; Bahcall 1979; De Grandi et al. 1999). Sample standard deviations $\mathrm{rms}_{\sigma}$ and $\mathrm{rms}_{L_{\mathrm{X}}}$ are $170 \mathrm{~km} \mathrm{~s}^{-1}$ and 0.3 dex for velocity dispersion and X-ray luminosity, respectively. As tested for a preliminary report (Marziani et al. 2013), no strong correlation emerges between prevalence of ELGs and the cluster velocity dispersion and virial radius in our sample (cf. Hwang et al. 2012). The WINGS clusters show a moderate dispersion in intrinsic properties that underlies the possibility of joining all clusters and forming a stacked sample of cluster galaxies.

\subsection{The galaxy sample of cluster members from WINGS - SPE (w1)}

The spectroscopic data are fully described by Cava et al. (2009). Here we recall the main features of the instrumental configurations used for WINGS spectroscopic survey (hereafter WINGS $\mathrm{SPE})$.

- Northern clusters: the $4.2 \mathrm{~m}$ William Herschel Telescope (WHT) was equipped with the AF2/WYFFOS multifiber spectrograph that yielded a spectral resolution customarily of $\approx 6 \AA$ or $\approx 3.2 \AA$ (for one observing run in October 2004) FWHM and 1.6 arcsec fiber diameter. Spectral coverage ranges from [OII] $\lambda 3727$ to [OI] $\lambda 6300$ for 1305 spectra of this sample sample.

\footnotetext{
1 https://sites.google.com/site/wingsomegawings/home
}

Table 1. Clusters belonging to WINGS considered in this study.

\begin{tabular}{|c|c|c|c|c|c|c|c|}
\hline Name & $z$ & $\begin{array}{c}\sigma \pm \delta \sigma \\
{\left[\mathrm{km} \mathrm{s}^{-1}\right]}\end{array}$ & $\begin{array}{c}\log L_{\mathrm{X}} \\
{\left[\mathrm{erg} \mathrm{s}^{-1}\right]}\end{array}$ & $\begin{array}{c}R_{200} \\
{[\mathrm{Mpc}]}\end{array}$ & $N_{1}$ & $N_{0}$ & $\mathrm{~N} / \mathrm{S}$ \\
\hline A1069 & 0.0653 & $690 \pm 68$ & 43.980 & 1.65422 & 40 & 72 & S \\
\hline A119 & 0.0444 & $862 \pm 52$ & 44.510 & 2.08724 & 158 & 90 & $S$ \\
\hline A151 & 0.0532 & $760 \pm 55$ & 44.000 & 1.83261 & 92 & 176 & S \\
\hline A1631a & 0.0461 & $640 \pm 33$ & 43.860 & 1.54845 & 125 & 99 & S \\
\hline A1644 & 0.0467 & $1080 \pm 54$ & 44.550 & 2.61226 & 176 & 90 & $S$ \\
\hline A 1831 & 0.0634 & $543 \pm 58$ & 44.280 & 1.30299 & 17 & 49 & $\mathrm{~N}$ \\
\hline A193 & 0.0485 & $759 \pm 59$ & 44.190 & 1.83428 & 39 & 22 & $\mathrm{~N}$ \\
\hline A1983 & 0.0447 & $527 \pm 38$ & 43.670 & 1.27589 & 45 & 49 & $\mathrm{~N}$ \\
\hline A1991 & 0.0584 & $599 \pm 57$ & 44.130 & 1.44081 & 35 & 15 & $\mathrm{~N}$ \\
\hline A 2107 & 0.0410 & $592 \pm 62$ & 44.040 & 1.43575 & 36 & 5 & $\mathrm{~N}$ \\
\hline A2124 & 0.0666 & $801 \pm 64$ & 44.130 & 1.91914 & 29 & 16 & $\mathrm{~N}$ \\
\hline A2169 & 0.0578 & $509 \pm 40$ & 43.650 & 1.22468 & 37 & 26 & $\mathrm{~N}$ \\
\hline A2382 & 0.0641 & $888 \pm 54$ & 43.960 & 2.13014 & 152 & 95 & $\mathrm{~S}$ \\
\hline A2399 & 0.0578 & $712 \pm 41$ & 44.000 & 1.71311 & 125 & 117 & $S$ \\
\hline A2415 & 0.0575 & $696 \pm 51$ & 44.230 & 1.67485 & 98 & 101 & $S$ \\
\hline A2457 & 0.0584 & $580 \pm 39$ & 44.160 & 1.39511 & 56 & 25 & $\mathrm{~N}$ \\
\hline A2572a & 0.0390 & $631 \pm 10$ & 44.010 & 1.53178 & 21 & 5 & $\mathrm{~N}$ \\
\hline A2589 & 0.0419 & $816 \pm 88$ & 44.270 & 1.97818 & 35 & 12 & $\mathrm{~N}$ \\
\hline A2593 & 0.0417 & $701 \pm 60$ & 44.060 & 1.69955 & 53 & 33 & $\mathrm{~N}$ \\
\hline A 2622 & 0.0610 & $696 \pm 55$ & 44.030 & 1.67205 & 38 & 33 & $\mathrm{~N}$ \\
\hline A2626 & 0.0548 & $625 \pm 62$ & 44.290 & 1.50593 & 36 & 34 & $\mathrm{~N}$ \\
\hline A 3128 & 0.0600 & $883 \pm 41$ & 44.330 & 2.12231 & 207 & 90 & $\mathrm{~S}$ \\
\hline A3158 & 0.0593 & $1086 \pm 48$ & 44.730 & 2.61110 & 177 & 101 & $\mathrm{~S}$ \\
\hline A 3266 & 0.0593 & $1368 \pm 60$ & 44.790 & 3.28911 & 225 & 39 & S \\
\hline A 3376 & 0.0461 & $779 \pm 49$ & 44.390 & 1.88475 & 92 & 52 & $\mathrm{~S}$ \\
\hline A 3395 & 0.0500 & $790 \pm 42$ & 44.450 & 1.90784 & 125 & 65 & $\mathrm{~S}$ \\
\hline A 3490 & 0.0688 & $694 \pm 52$ & 44.240 & 1.66101 & 83 & 135 & $\mathrm{~S}$ \\
\hline A3497 & 0.0680 & $726 \pm 47$ & 44.160 & 1.73827 & 82 & 83 & S \\
\hline A3556 & 0.0479 & $558 \pm 37$ & 43.970 & 1.34890 & 114 & 61 & $\mathrm{~S}$ \\
\hline A 3560 & 0.0489 & $710 \pm 41$ & 44.120 & 1.71554 & 117 & 73 & $S$ \\
\hline A376 & 0.0476 & $852 \pm 49$ & 44.140 & 2.05991 & 66 & 22 & $\mathrm{~N}$ \\
\hline A3809 & 0.0627 & $563 \pm 40$ & 44.350 & 1.35144 & 104 & 91 & $\mathrm{~S}$ \\
\hline A500 & 0.0678 & $658 \pm 48$ & 44.150 & 1.57561 & 89 & 51 & $\mathrm{~S}$ \\
\hline A671 & 0.0507 & $906 \pm 58$ & 43.950 & 2.18725 & 20 & 15 & $\mathrm{~N}$ \\
\hline A754 & 0.0547 & $1000 \pm 48$ & 44.900 & 2.40961 & 126 & 25 & $\mathrm{~S}$ \\
\hline A957x & 0.0451 & $710 \pm 53$ & 43.890 & 1.71862 & 65 & 62 & $\mathrm{~S}$ \\
\hline A970 & 0.0591 & $764 \pm 47$ & 44.180 & 1.83708 & 116 & 67 & $\mathrm{~S}$ \\
\hline IIZW108 & 0.0483 & $513 \pm 75$ & 44.340 & 1.23989 & 27 & 4 & $\mathrm{~S}$ \\
\hline MKW3s & 0.0444 & $539 \pm 37$ & 44.430 & 1.30513 & 32 & 34 & $\mathrm{~N}$ \\
\hline RX0058 & 0.0484 & $637 \pm 97$ & 43.640 & 1.53951 & 20 & 8 & $\mathrm{~N}$ \\
\hline RX1022 & 0.0548 & $577 \pm 49$ & 43.540 & 1.39028 & 25 & 19 & $\mathrm{~N}$ \\
\hline RX1740 & 0.0441 & $582 \pm 65$ & 43.700 & 1.40944 & 20 & 12 & $\mathrm{~N}$ \\
\hline Z2844 & 0.0503 & $536 \pm 53$ & 43.760 & 1.29425 & 33 & 21 & $\mathrm{~N}$ \\
\hline Z8338 & 0.0494 & $712 \pm 60$ & 43.900 & 1.71996 & 53 & 33 & $\mathrm{~N}$ \\
\hline Z8852 & 0.0408 & $765 \pm 63$ & 43.970 & 1.85550 & 53 & 18 & $\mathrm{~N}$ \\
\hline
\end{tabular}

- Southern clusters: the $3.9 \mathrm{~m}$ Anglo Australian Telescope (AAT) was equipped the $2 \mathrm{dF}$ multifiber spectrograph that yielded a spectral resolution of $9 \AA$ FWHM and a 2 arcsec fibre diameter. Coverage extends from [OII] $\lambda 3727$ to [SII] $\lambda \lambda 6716,6731$.

Spectrophotometric data are available for 5859 sources in the fields of 46 of the original 77 clusters covered by the survey. Of these, 3514 spectra were of cluster members. All cluster member spectra were then joined to form a stacked sample with 3514 spectra. The exact number of cluster sources considered in the following analysis varies depending on the availability of morphological and photometric parameters in the WINGS database. The remaining 2345 were field galaxies and allowed the extraction of suitable control samples matching cluster members in luminosity and morphological type (Sect. 2.3).

Morphological type, luminosity, colors, and structural parameters for most of the galaxies of the present samples were retrieved from the public WINGS database (Moretti et al. 2014; Fasano et al. 2012; D'Onofrio et al. 2014). 


\subsection{Non-cluster galaxies (w0) and control samples}

Spectroscopic targets were selected from the available WINGS optical $B$ and $V$ photometry (Varela et al. 2009). The spectroscopic survey criteria were defined to maximize the chances of observing galaxies at the cluster redshift without biasing the cluster sample (for a detailed description, see Cava et al. 2009; and Fritz et al. 2014). The ratio between the sources spectroscopically confirmed to belong to a cluster $N_{1}$ with respect to the spectroscopic observations for that cluster, i.e., $N_{1} /\left(N_{1}+N_{0}\right)$ (where $N_{0}$ is the number of sources with redshift not consistent with cluster membership following Cava et al. 2009) is significantly less than one, as evident from the columns of Table 1 that list $N_{0}$ and $N_{1}$. This means that a sizable sample of non-cluster galaxies can be defined. This sample (hereafter denoted also as the w0 sample, or with the subscript 0 ) has been observed with exactly the same instrumental setup used for targets that turned out to be cluster members (the w1 sample, subscript 1), making it well suited for the definition of a control sample (CS). The w1 sample is a stacked sample that include all 46 cluster spectra. Approximately $170 \mathrm{w} 0$ galaxies most likely belong to background clusters. They were identified from the detection of a second red sequence in the color-magnitude diagrams of Valentinuzzi et al. (2011) displaced from the red sequence of the WINGS cluster, and removed from the w0 sample.

The redshift distribution indicates that the $\mathrm{w} 0$ galaxies are mainly background galaxies in the redshift range $0.01 \lesssim z \lesssim 0.3$, with distribution mode at $z \approx 0.15$, while the cluster galaxies are distributed over a narrow range of redshift $(0.04 \lesssim z \lesssim$ $0.08)$. Since galaxy emission line properties strongly depend on absolute magnitude $M_{V}$ and morphological type, the stacked sample of non-cluster galaxies w0 cannot be compared to the stacked cluster sample if systematic differences in, for example, ELGs prevalence and emission line luminosity, are under scrutiny. The distributions of absolute magnitude, morphological type (de Vaucouleurs' $t$ ), and the ratio between the fiber diameter $d_{\text {fib }}$ and $R_{\mathrm{e}}$ measured in arcsec for non-cluster galaxies are different from those of cluster members (Fig. 1), even if evolutionary effects should be relatively minor. The Madau plot (Madau et al. 1998; Madau \& Dickinson 2014) shows that evolutionary effects are expected to increase at most the star formation rate by $\delta \log S F R \approx 0.25$ in the range $0.05 \leq z \leq 0.3$.

Non-cluster galaxies still provide a pooling sample from which control samples matching the luminosity and morphological type distribution of the stacked clusters samples are extracted. We constructed a large number $\left(\sim 10^{3}\right)$ of CSs extracted semi-randomly (as described below) from the stacked sample of non-member galaxies to overcome systematic differences in $M_{V}$, $R_{\mathrm{e}}$, and $t$. Non-cluster members were randomly selected in $M_{V}$ intervals with a distribution that mimics the differential $M_{V}$ distribution for cluster galaxies. We then imposed the condition that the $M_{V}$ and either the $R_{\mathrm{e}}$ or $t$ distributions are not statistically distinguishable from the cluster member distribution at a confidence level larger than $3 \sigma$ according to the $D$ estimator of the Kolmogorov-Smirnov test. The de Vaucouleurs' morphological parameter $t$ is treated as a continuous variable in this context.

Figure 1 shows the cumulative and differential distributions of $t, M_{V}, \log d_{\mathrm{fib}} / R_{\mathrm{e}}$ with $d_{\mathrm{fib}}=2^{\prime \prime}$ and $1.6^{\prime \prime}$ (for the southern and northern sample, respectively) for the full sample and cluster and non-member galaxies (top panels), for the full sample of cluster galaxies and one realization of the CSs matching $t$ and $M_{V}\left(\mathrm{w} 0_{\mathrm{t}, \mathrm{M}}\right.$, second row from top), and one matching $\log \left(d_{\mathrm{fib}} / R_{\mathrm{e}}\right)$ and $M_{V}\left(\mathrm{w} 0_{\mathrm{t}, \mathrm{R}}\right.$, third row $)$.

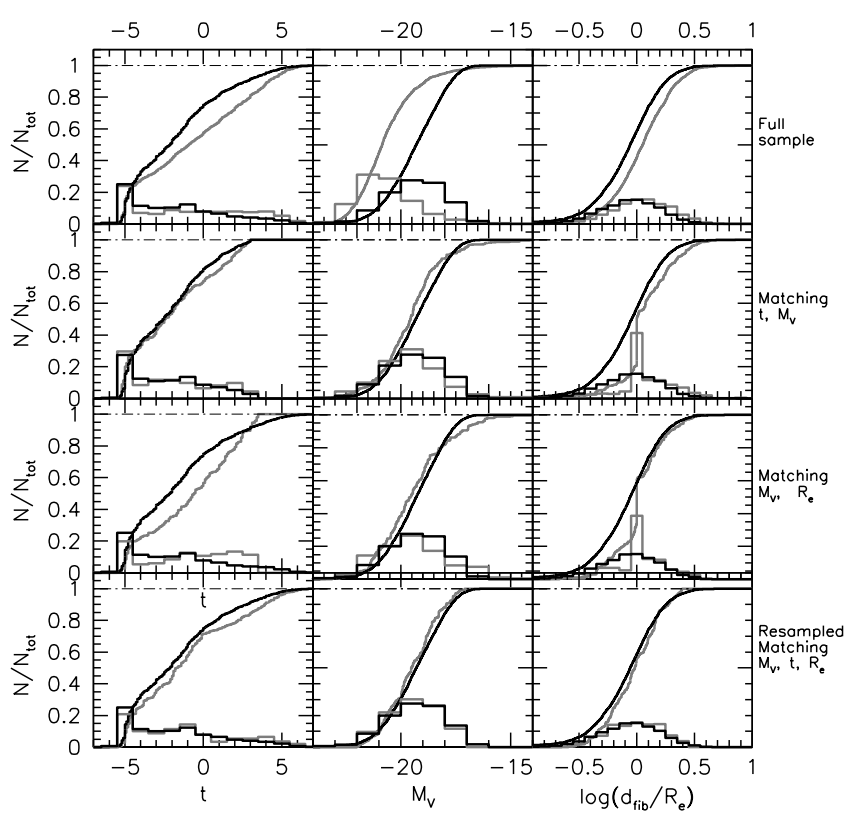

Fig. 1. Cumulative and differential distribution of de Vaucouleurs't (left panel), of $M_{V}$ (middle), and ratio aperture to effective radius $\log \left(d_{\mathrm{fib}} / R_{\mathrm{e}}\right)$, for cluster members (black) and non-cluster galaxies (gray). Top row of panels: all cluster and non-cluster galaxies included in the present study (i.e., w1 and w0 samples); second row of panels from top: cluster member vs. CS with matched distributions of $M_{V}$ and de Vaucouleurs' $t\left(\mathrm{wO}_{\mathrm{t}, \mathrm{M}}\right)$; third row from top: cluster member vs. CS with matched distributions of $R_{\mathrm{e}}$ and de Vaucouleurs' $t\left(\mathrm{w}_{\mathrm{t}, \mathrm{R}_{\mathrm{e}}}\right)$. Bottom row: resampled CS matching $M_{V}, t$, and $R_{\mathrm{e}}\left(\overline{\mathrm{w}}_{\mathrm{t}, \mathrm{M}, \mathrm{R}}\right)$.

Extraction of control samples following the procedure described above has several advantages and drawbacks.

- The cluster sample is preserved almost in full. There are limitations introduced to allow for adequate sampling of the w0 parameter space: for $\mathrm{w}_{\mathrm{t}, \mathrm{M}}, M_{V} \geq-23.5, t \leq 3$., $\log \left(d_{\mathrm{fib}} / R_{\mathrm{e}}\right) \leq 1.5$; for $\mathrm{w} 0_{\mathrm{t}, \mathrm{R}_{\mathrm{e}}}, M_{V} \geq-23 ., t \leq 3.5$, $\log \left(d_{\mathrm{fib}} / R_{\mathrm{e}}\right)$ unconstrained. The number of sources is $\approx 3000$ in both cases.

- Biases and selection effects are not corrected for, but control samples are meant to reproduce the same biases and selection effects operating on cluster galaxies. The CSs are however not meant to be representative of a field galaxy population.

- The $3 \sigma$ conditions are fairly restrictive. Samples are large so that a $3 \sigma$ confidence level is reached when differences between samples do not introduce a significant bias as shown in Fig. 1.

- The semi-random extraction procedure makes possible the realization of a large number of control samples that are used for bootstrap estimates of significance (Appendix A).

On the other hand, it proved difficult to extract very large control samples since we could extract at most samples of $\approx 300$ sources. In the following we consider mainly the control samples defined by matching $t$ and $M_{V}$. In this case the median $R_{\mathrm{e}}$ for cluster and non-member samples differ by $\delta \log \left(d_{\text {fib }} / R_{\mathrm{e}}\right) \approx 0.2$, implying a factor of $\approx 2.5$ in the galaxy surface sampled. An ideal control sample would have conditions of statistical indistinguishability concurrently satisfied for $t, M_{V}$, and $\log R_{\mathrm{e}}$. Therefore, an additional control sample has been defined on the basis of a completeness approach. We considered a 3D parameter space with axes $M_{V}, R_{\mathrm{e}}$, and $t$, binned in steps $\Delta$ for each variable. The parameter space volume bins $\Delta V=\Delta M_{V} \Delta t \Delta R_{\mathrm{e}}$ were chosen 
so that at least two sources were present for the non-member. We then considered weighting factors for the w0 sample sources given by

$\beth\left(M_{V}, t, R_{\mathrm{e}}\right)=\frac{N_{1}\left(M_{V}, t, R_{\mathrm{e}}\right)}{N_{0}\left(M_{V}, t, R_{\mathrm{e}}\right)}, \forall \Delta V \neq \emptyset$.

Computing this correction factor is relatively straightforward. The method has the significant drawback that $\mathrm{J}\left(M_{V}, t, R_{\mathrm{e}}\right)$ becomes $\gg 1$ if there are few w0 sources and many cluster sources within a given $\Delta V$. The range in the parameter space is $-17 \geq M_{V} \geq-23.5$ (sources fainter than $M_{V} \approx-17$ are almost completely absent $), t$ unconstrained, and $\log \left(d_{\mathrm{fib}} / R_{\mathrm{e}}\right) \leq 1.4$. The results of one realization of the resampled CSs (hereafter indicated as $\left.\overline{\mathrm{w}}_{\mathrm{t}, \mathrm{M}, \mathrm{R}}\right)$ are shown in the bottom panel of Fig. 1.

\section{Measurements and data analysis}

\subsection{Spectral modeling}

Faint emission lines ( $W \lesssim 1 \AA$ ) are superposed to the stellar population spectra in a large number of WINGS spectra. They are unresolved and often fully buried within the absorption associated with the quiescent stellar population. Sources with strong emission lines ( $W \gtrsim 3 \AA$ ) are relatively rare (Sect. 4.3). In a minority of cases the continuum raises toward the blue, and emission lines are strong and consistent with high excitation HII emission or with type 2 nuclear activity. Only in two cases we found a type 1.0 spectrum (Sect. 3.2).

Spectral models with synthetic stellar populations were computed by Fritz et al. (2011) for a large part of WINGS - SPE galaxies. Sources without spectral modeling from Fritz et al. (2011) were analyzed using Bruzual \& Charlot (2003) population synthesis templates. The best fit was achieved by $\chi^{2}$ minimization techniques using the IRAF task SPECFIT (Kriss 1994), which allowed each fit to be carried out interactively.

\subsection{Line intensity, line ratios, and equivalent widths}

The typical instrumental properties of WINGS - SPE and the characteristics of the observed spectra justify an approach in which each spectrum is analyzed as the sum of stellar emission and faint emission lines whose width is dominated by the instrumental profile and is not changing significantly from line to line. The measurement procedure was devised accordingly. The emission components of relevant lines ([OII] $\lambda 3727$, $\mathrm{H} \beta,[\mathrm{OIII}] \lambda \lambda 4959,5007,[\mathrm{OI}] \lambda 6300, \mathrm{H} \alpha,[\mathrm{NII}] \lambda 6583$, and [SII] $\lambda \lambda 6716,6731)$ were obtained by subtracting underlying stellar emission computed by population synthesis; examples of these emission components are shown in Fig. 2. Since population synthesis models often do not satisfactorily reproduce the whole spectrum from 3700 to $7000 \AA$, we applied a two-step procedure to ensure that maximum accuracy is obtained for the $\mathrm{H} \beta$ spectral range. A first fit was carried out by SPECFIT on a broad range of wavelengths, typically of 3800-5500 $\AA$, where exact values are dependent on the rest-frame spectral coverage and presence of zapped regions or contaminated by spikes. A second fit was carried out by restricting the spectral range to the $\mathrm{H} \beta$ region, typically between 4600 and $5100 \AA$, to ensure minimization of any residual underlying $\mathrm{H} \beta$. This second fit required only minor adjustment in the scaling factor, but it proved to be necessary since the overall best fit did not always yield the minimum $\chi^{2}$ in the $\mathrm{H} \beta$ spectral region. A small (few tenths of $\AA$ ) wavelength adjustment also proved to be necessary in some cases. Intensity measures were carried out on the residual spectra, i.e., on the original, deredshifted galaxy spectra minus the scaled population synthesis
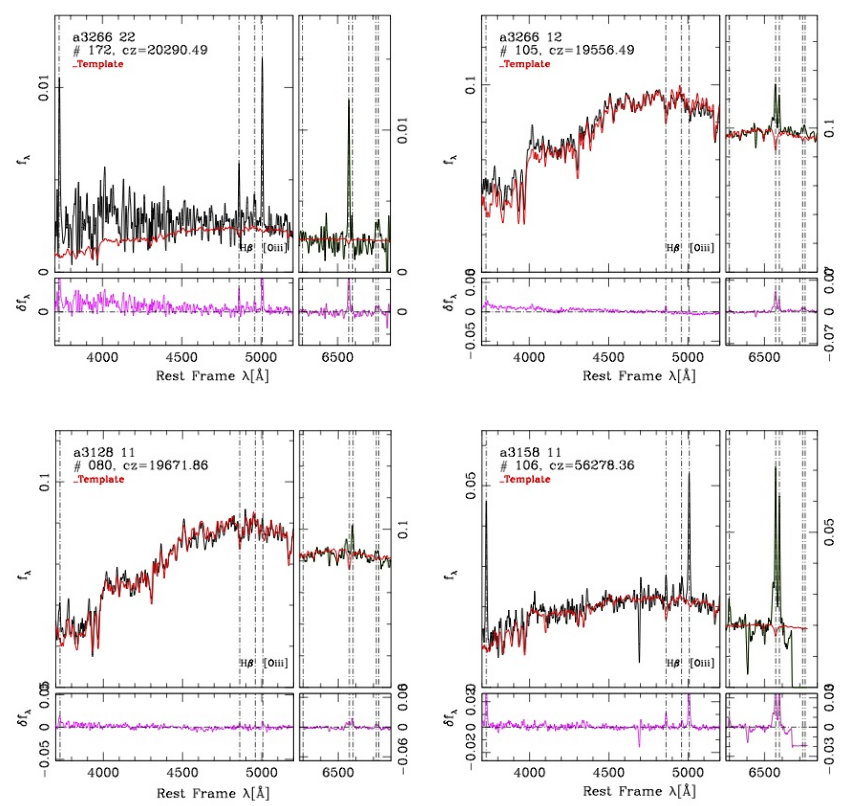

Fig. 2. Examples of ELG spectra. Top left: the high-ionization HII galaxy WINGS J043319.43-614141.8; the TO WINGSJ043021.56612848.7; bottom left: the LINER WINGSJ032933.77-522659.6; bottom right the Seyfert 2 galaxy WINGSJ034144.52-534221.1. Red lines trace the adopted stellar template. All of these sources could be entered in the DD with detected diagnostic ratios and have the probability of proper classification $P_{i, i} \gtrsim 0.8$.

model. A second background subtraction was performed on the emission lines in the residual spectra. The intensity measure was taken as the peak value of the line within a fixed window in the residual spectra.

Problematic aspects involved an inaccurate wavelength scale calibration shortward of $4000 \AA$ because of the lack of reference lines from lamp and sky. The [OII] $\lambda 3727$ line often appeared displaced with respect to the window used for the automatic intensity line measure. In such cases, the line was measured interactively by the IRAF task SPLOT.

An atlas showing the galaxy spectrum, the population synthesis model, and the residual was created for all the spectra analyzed. Four examples are shown in Fig. 2. The atlas was visually inspected and cross-checked against the measures to ensure the rejection of cases with spike contamination and bad data and the identification of cases that did not meet the assumption on which this procedure is based: sources with broad lines $\left(\gtrsim 1000 \mathrm{~km} \mathrm{~s}^{-1}\right.$, as expected for type 1) and a mixture of host and non-thermal emission. As mentioned, such sources proved to be very rare: three cases that belong to clusters and four cases that do not belong to clusters (their identification is reported in Sect. 4.3).

\subsection{Criteria for the identification of quiescent and emission line galaxies}

The following selection criterion was adopted to identify sources with emission lines:

$$
\begin{aligned}
& \{[\mathrm{OII}] \text { AND }[[\mathrm{OIII}] \text { OR } \mathrm{H} \beta]\} \\
& \text { OR } \\
& \{([\mathrm{OIII}] \mathrm{OR} \mathrm{H} \beta) \text { AND }([\mathrm{NII}] \mathrm{OR} \mathrm{H} \alpha)\},
\end{aligned}
$$


where a line was considered detected if $I_{\mathrm{p}} / \mathrm{rms}$ greater than 3 , where $I_{\mathrm{p}}$ is the ratio between peak intensity relative to background and local noise as measured from the rms scatter in a wavelength range adjacent to the line in consideration. The detection limit on $I_{\mathrm{p}} / \mathrm{rms}$ has been set to 4 to create a subset of higher quality measures. The criterion can be applied to almost all spectra of the northern and southern samples but does not ensure the ability to place each source with detected emission lines in the canonical diagnostic diagrams. This criterion has the considerable advantage that it is less biased than criteria based on a minimum equivalent width of the Balmer lines. Selecting sources on the basis of a minimum equivalent width in $\mathrm{H} \beta(\geq 3 \AA$ was used in the preliminary analysis of Marziani et al. 2013) clearly biases the selection of ELGs in favor of star-forming systems, and may exclude a population of low-luminosity ELGs (for example, low EW sources with [OIII] $\lambda 5007 / \mathrm{H} \beta>1$ and $[\mathrm{NII}] \lambda 6583 / \mathrm{H} \alpha>1$ ) that may not be associated with HII sources. The analysis of errors on line intensity and line ratios, and censored data is described in Appendix A.1.

\subsection{Line flux, luminosity, and SFR estimates}

Aperture Johnson magnitudes $V^{\prime}$ and $B^{\prime}$ (at fixed width 2 arcsec) were corrected for Galactic extinction (with $A_{V}$ and $A_{B}$ retrieved from NED) and $k$-corrected following Poggianti (1997). The line rest-frame luminosity can be obtained from the line flux $F$ derived from the aperture magnitudes as in the following equation:

$L=4 \pi D_{\mathrm{L}}^{2} W f_{\lambda} 10^{0.4\left(V^{\prime}-V_{\text {best }}^{\prime}\right)}$,

where $D_{\mathrm{L}}$ is the luminosity distance and $W$ is the line equivalent width. The continuum flux $f_{\lambda}$ was computed from the average of $f_{\lambda, V}=f_{\lambda_{V, 0}} 10^{-0.4 V^{\prime}}$ and $f_{\lambda, B}=f_{\lambda_{B, 0}} 10^{-0.4 B^{\prime}}$ for $\mathrm{H} \beta$ where $f_{\lambda_{V, 0}}$ and $f_{\lambda_{V, 0}}$ are the 0 -point fluxes for the $B$ and $V$ bands, respectively, and from $f_{\lambda, V}$ only for $\mathrm{H} \alpha$. The last factor is the correction for aperture ( $V_{\text {best }}^{\prime}$ is the total isophotal magnitude of the galaxy, cf. Hopkins et al. 2003, for $\mathrm{H} \alpha$ ), and assumes that the line flux measured within a fixed angular aperture can be scaled by the ratio of the total galaxy flux and of the galaxy flux within the aperture. Estimates of line fluxes and luminosities are therefore expected to be subject to a large uncertainty.

The star formation rate (SFR) following Kennicutt (1998) is

$S F R=\frac{L(\mathrm{H} \alpha)}{\eta_{\mathrm{H} \alpha}} M_{\odot} \mathrm{yr}^{-1}=\frac{L(\mathrm{H} \beta)}{\eta_{\mathrm{H} \beta}} M_{\odot} \mathrm{yr}^{-1}$,

where $\eta_{\mathrm{H} \alpha} \approx 1.27 \times 10^{41} M_{\odot} \mathrm{yr}^{-1} \mathrm{erg}^{-1} \mathrm{~s}$, with $\eta_{\mathrm{H} \alpha}=2.85 \eta_{\mathrm{H} \beta}$. No correction for internal extinction was applied.

\section{Results}

\subsection{The catalog for the WINGS database}

Relevant quantities extracted from our measurements and analysis are reported in a table appended to the paper in machinereadable form. The descriptions of each individual record are provided in Table B.1. Here entries are briefly discussed to make their overall meaning explicit.

- $\mathrm{S} / \mathrm{N}$ - The noise measurement is the rms in the spectral range 5030-5060 $\AA$, and the $\mathrm{S} / \mathrm{N}$ is computed by taking the continuum in correspondence of [OIII] $\lambda 5007$ as signal.
- Ratio peak intensity to noise - The ratio peak intensity over local noise $I_{\mathrm{p}} / \mathrm{rms}$ is reported for all measured lines. This parameter is used for establishing ELG detection following Eq. (2) and for distinguishing between detections (flag 0 ) and non-detections in the individual lines (upper limits, flag -1). The $I_{\mathrm{p}} / \mathrm{rms}$ can be used as surrogate of line intensity for nearby lines in which the rms noise can be considered constant. Intensity ratios based one line in the $\mathrm{H} \beta$ and one in the $\mathrm{H} \alpha$ range should be viewed with care because of the uncertain relative spectrophotometric calibration: high $\mathrm{S} / \mathrm{N}$ spectra often yield $I_{\mathrm{p}}(\mathrm{H} \alpha) / I_{\mathrm{p}}(\mathrm{H} \beta) \approx 2$.

- Equivalent widths - Rest-frame equivalent widths (after stellar absorption correction) are reported in $\AA$. Errors for detection were estimated by propagating error on flux and on continuum measures. If the measured equivalent width is below the minimum detectable value computed as a function of $\mathrm{S} / \mathrm{N}$, a censorship flag [EW_HB_CENSOR] is set to -1 . The data are optimized for the $\mathrm{H} \beta-[\mathrm{OIII}] \lambda \lambda 4959,5007$ spectral range. $W(\mathrm{H} \beta)$ should be usually preferred over $W(\mathrm{H} \alpha)$. Even if the $\mathrm{H} \alpha$ spectral range is covered in the Southern sample spectra, the observations are not optimized at $\mathrm{H} \alpha$. In several cases the continuum goes down to 0 , leading to a divergent $W(\mathrm{H} \alpha)$ value. This is turn affects $\mathrm{H} \alpha$ luminosity and SFR estimates based on $\mathrm{H} \alpha$.

- Diagnostic ratios - We report values, uncertainties, and censorship flags for the five diagnostic ratios [OIII] $\lambda 5007 / \mathrm{H} \beta$, $[\mathrm{NII}] \lambda 6583 / \mathrm{H} \alpha, \quad[\mathrm{OII}] \lambda 3727 / \mathrm{H} \beta, \quad[\mathrm{OI}] \lambda 6300 / \mathrm{H} \alpha$, and [SII] $\lambda \lambda 6716,6731 / \mathrm{H} \alpha$. In case of detections (censorship flag 0), errors were computed following Rola \& Pelat (1994) on the basis of the $I_{\mathrm{p}} / \mathrm{rms}$ values of each line. Upper limits (censorship flag -1) and lower limits (censorship flag 1) have no associated uncertainty; non-availability is coded as -999 and the code -888 identifies cases in which the spectral range is covered but the ratio cannot be computed because both lines are undetected.

- Classification and probability of classification - We report classification (HII, LINER, Seyfert, and TO coded as EH, EL, ES, and ET) for sources with detected emission lines and for sources whose emission line ratios allowed us to place a data point in the diagnostic diagram [OIII] $\lambda 5007 / \mathrm{H} \beta$ versus $[\mathrm{NII}] \lambda 6583 / \mathrm{H} \alpha$ (hereafter $\mathrm{DD}_{[\mathrm{NII}]}$ ). The data point can be associated with a detection in two diagnostic ratios, or with lower and upper limits in one or both diagnostic ratios. For each source in region $i$ (for $i=\mathrm{EH}, \mathrm{ET}, \mathrm{EL}, \mathrm{ES}$ ), a probability of correct classification $P_{i, i}$ is reported along with the probability that the source may be misclassified $\left(P_{i, j}\right.$, with $\left.i \neq j\right)$ and the correct classification is one of the remaining classes. A revised classification code is assigned on the basis of the ELG class if the highest probability of correct classification occurs in a region different from the original classification region, i.e., if $P_{i, j}>P_{i, i}$. The revised classification code was only assigned for the diagnostic diagram [OIII] $\lambda 5007 / \mathrm{H} \beta$ versus [OII $] \lambda 3727 / \mathrm{H} \beta$ (hereafter $\left.\mathrm{DD}_{[\mathrm{OII}]}\right)$ and $\mathrm{DD}_{[\mathrm{NII}]}$, and only the latter was used in the analysis presented in this paper. The classifications are reported for the four DDs. However, in examining individual sources, a spectral type should be assigned on the basis of $\mathrm{DD}_{[\mathrm{NII}]}$, which overrules the other DDs. In the case in which $\mathrm{DD}_{[\mathrm{NII}]}$ is not available because the $\mathrm{H} \alpha$ range is not covered (Northern sample), then $\mathrm{DD}_{[\mathrm{OII}]}$ can be used with [OI] $\lambda 6300 / \mathrm{H} \beta$ to test the possibility of a misclassified LINER, TO, or Seyfert. The LINER WINGSJ032933.77-522659.6 and the Seyfert 2 galaxy WINGSJ034144.52-534221.1 of Fig. 2 are classified as $\mathrm{HII}$ in the $\mathrm{DD}_{[\mathrm{OII}]}$ diagram. Individual source 
Table 2. Counts of galaxies assigned to different classes from the $\mathrm{DD}_{[\mathrm{NII}]}$.

\begin{tabular}{|c|c|c|c|c|c|c|c|c|c|c|c|c|c|c|}
\hline Sample & $W_{\min }$ & $N$ & $N_{\mathrm{U}}$ & $N_{\mathrm{E}}$ & $N_{\mathrm{DD}}$ & $N_{\text {DD,d }}$ & $N_{\mathrm{HII}}$ & $N_{\mathrm{TO}}$ & $N_{\text {Lin }}$ & $N_{\text {Seyf }}$ & $N_{\text {HII,rev }}$ & $N_{\text {TO,rev }}$ & $N_{\text {lin,rev }}$ & $N_{\text {seyf,rev }}$ \\
\hline \multicolumn{15}{|c|}{$(S / N)_{\min }:$ no restriction, detection following Eq. (2), $I_{\mathrm{p}} / \mathrm{rms}>3$} \\
\hline w1 & -999 & 3514 & 2611 & 903 & 801 & 414 & 323 & 58 & 22 & 11 & 330 & 52 & 18 & 14 \\
\hline w0 & -999 & 2345 & 993 & 1352 & 863 & 448 & 342 & 88 & 6 & 12 & 366 & 63 & 7 & 12 \\
\hline \multicolumn{15}{|c|}{$(S / N)_{\min }=3$, detection following Eq. $(2), I_{\mathrm{p}} / \mathrm{rms}>3$} \\
\hline w1 & -999 & 3461 & 2567 & 894 & 793 & 413 & 322 & 58 & 22 & 11 & 329 & 52 & 18 & 14 \\
\hline w1 & 3 & 448 & 0 & 448 & 448 & 319 & 304 & 9 & 0 & 6 & 304 & 9 & 0 & 6 \\
\hline w0 & -999 & 2211 & 926 & 1285 & 818 & 434 & 330 & 84 & 6 & 14 & 352 & 61 & 7 & 14 \\
\hline w0 & 3 & 645 & 0 & 645 & 645 & 384 & 316 & 60 & 0 & 8 & 335 & 41 & 0 & 8 \\
\hline $\mathrm{w}_{\mathrm{t}, \mathrm{M}}$ & -999 & 371 & 144 & 227 & 170 & 96 & 81 & 13 & 1 & 1 & 83 & 11 & 1 & 1 \\
\hline $\mathrm{w} 0_{\mathrm{t}, \mathrm{M}}$ & 3 & 147 & 0 & 147 & 147 & 94 & 81 & 12 & 0 & 1 & 83 & 10 & 0 & 1 \\
\hline$\overline{\mathrm{w}}_{\mathrm{t}, \mathrm{M}, \mathrm{R}}$ & -999 & 2471 & 1209 & 1262 & 977 & 554 & 487 & 45 & 1 & 21 & 502 & 29 & 2 & 21 \\
\hline$\overline{\mathrm{w}}_{\mathrm{t}, \mathrm{M}, \mathrm{R}}$ & 3 & 756 & 0 & 756 & 756 & 509 & 478 & 25 & 0 & 6 & 493 & 10 & 0 & 6 \\
\hline \multicolumn{15}{|c|}{$(S / N)_{\min }=4$, detection following Eq. (2), $I_{\mathrm{p}} / \mathrm{rms}>4$} \\
\hline w1 & -999 & 3461 & 2799 & 662 & 607 & 299 & 253 & 25 & 11 & 10 & 253 & 26 & 11 & 9 \\
\hline w1 & 3 & 421 & 0 & 421 & 421 & 260 & 246 & 8 & 0 & 6 & 246 & 8 & 0 & 6 \\
\hline w0 & -999 & 2211 & 1120 & 1091 & 701 & 319 & 257 & 50 & 3 & 9 & 269 & 40 & 1 & 9 \\
\hline w0 & 3 & 606 & 0 & 606 & 606 & 295 & 250 & 38 & 0 & 7 & 261 & 27 & 0 & 7 \\
\hline $\mathrm{wO}_{\mathrm{t}, \mathrm{M}}$ & -999 & 351 & 163 & 188 & 130 & 67 & 53 & 12 & 0 & 2 & 55 & 10 & 0 & 2 \\
\hline $\mathrm{w} 0_{\mathrm{t}, \mathrm{M}}$ & 3 & 116 & 0 & 116 & 116 & 62 & 53 & 7 & 0 & 2 & 55 & 5 & 0 & 2 \\
\hline$\overline{\mathrm{w}}_{\mathrm{t}, \mathrm{M}, \mathrm{R}}$ & -999 & 2471 & 1395 & 1076 & 838 & 371 & 330 & 33 & 0 & 8 & 341 & 22 & 0 & 8 \\
\hline $\mathrm{W}_{\mathrm{t}, \mathrm{M}, \mathrm{R}}$ & 3 & 732 & 0 & 732 & 732 & 345 & 323 & 19 & 0 & 3 & 334 & 8 & 0 & 3 \\
\hline
\end{tabular}

classifications only make sense only if both diagnostic ratios are uncensored or for HIIs whose ratios [OIII] $\lambda 5007 / \mathrm{H} \beta$ and/or [OII] $\lambda 3727 / \mathrm{H} \beta$ and $[\mathrm{NII}] \lambda 6583 / \mathrm{H} \alpha$ are upper limits (which implies $P_{i, i}=1$ ). Otherwise, classification based on censored line ratios have only statistical value (with the caveats of Appendix A.1) and, for individual sources, should be confirmed by additional observations.

- Flux and luminosity - We report specific flux per unit wavelength at $5000 \AA$ in units of $\operatorname{erg~s}^{-1} \mathrm{~cm}^{-2} \AA^{-1}$, and at the effective wavelength of the $V$ band. Owing to the tentative nature of flux estimates (Sect. 3.4), we do not assign an error to specific continuum fluxes. The $\mathrm{H} \alpha$ and $\mathrm{H} \beta$ luminosity are also reported without error assignment. For these quantities only a detection or censorship flag is given.

- Notes on individual sources - The notes identify type 1 sources and sources that belong to background clusters. A comment is added in some cases of instrumental, calibration, or fitting problems and in the cases in which a large redshift correction was needed.

\subsection{Prevalence of quiescent and emission-line galaxies}

Table 2 lists the number of sources for the full samples of cluster and non-member galaxies without restriction on $S / N$ (top), $S / N_{\min }=3$ (middle), and $S / N_{\min }=4$ (bottom). The code number -999 is used to indicate that all sources are included, i.e., that there is no restriction on $W(\mathrm{H} \beta)$. The first column reports the samples (w1: cluster member, $\mathrm{w} 0$ : non-member, $\mathrm{w}_{\mathrm{t}, \mathrm{M}}, \overline{\mathrm{w}}_{\mathrm{t}, \mathrm{M}, \mathrm{R}}$ ). The second column lists the assumed minimum $W(\mathrm{H} \beta)$ in emission. Column 3 provides the total number of sources and Col. 4 $\left(N_{\mathrm{U}}\right)$ lists the number of sources with non-detection. The numbers of sources with detected emission lines are listed in Col. 5 $\left(N_{\mathrm{Em}}\right)$. Column 6 provides the number of sources that could be placed in the $\mathrm{DD}_{[\mathrm{NII}]}\left(N_{\mathrm{DD}}\right)$. The following columns list the numbers of HII $\left(N_{\mathrm{H}}\right)$, TO $\left(N_{\mathrm{T}}\right)$, LINER $\left(N_{\mathrm{L}}\right)$, and Seyfert $\left(N_{\mathrm{S}}\right)$ sources, before and after classification revision using $\mathrm{DD}_{[\mathrm{NII}]}$
(Sect. A.1). The counts of Table 2 refer to diagnostic ratios computed from detected emission lines. The counts with censored diagnostic ratios (which can be retrieved from the digital table described in Sect. 4.1) yield consistent results.

\subsection{A deficit of ELGs in the clusters of WINGS - SPE}

The prevalence of ELGs is measured by the ratio $f_{\mathrm{Em}}=$ $N_{\mathrm{Em}} / N=N_{\mathrm{Em}} /\left(N_{\mathrm{U}}+N_{\mathrm{Em}}\right)$, or by $R_{\mathrm{Em}}=N_{\mathrm{Em}} / N_{\mathrm{U}}=\left(N_{\mathrm{H}}+\right.$ $\left.N_{\mathrm{N}}+N_{\mathrm{E}}\right) / N_{\mathrm{U}}$. Here $N_{\mathrm{E}}$ is the number of ELGs with detected emission lines but insufficient data for DD classification, and $N_{\mathrm{N}}$ is the number of ELGs that do not show an HII spectrum, i.e., $N_{\mathrm{N}}=N_{\mathrm{L}}+N_{\mathrm{S}}+N_{\mathrm{TO}}=N_{\mathrm{TO}}+N_{\mathrm{A}}$, where $N_{\mathrm{A}}$ is the number of AGNs $\left(=N_{\mathrm{L}}+N_{\mathrm{S}}\right)$. The ratios $f_{\mathrm{Em}}$ and $R_{\mathrm{Em}}$ are different among cluster members and non-cluster members (Table 2). The pie diagrams of Fig. 3 graphically show a clear deficit of ELGs in clusters. This result holds with respect to the $\mathrm{w}_{\mathrm{t}, \mathrm{M}}, \mathrm{w} 0_{\mathrm{t}, \mathrm{R}}$, and $\overline{w 0}_{\mathrm{t}, \mathrm{M}, \mathrm{R}}$ and with respect to the $\mathrm{w} 0$ sample, confirming the preliminary results from a subsample of 1305 WINGS - SPE sources (Marziani et al. 2013). The AGNs (LINERs + Seyferts) appear to be rare in both cluster and non-cluster samples, with prevalence $f_{\mathrm{A}} \approx 3 \%$. The TO population is sizable in $\mathrm{w} 1$ and control samples (Table 2). The ratio $R_{\mathrm{T}}=N_{\mathrm{TO}} / N_{\mathrm{H}}$ for revised classes is $\approx 16 \%$ in $\mathrm{w} 1$ and $\approx 5 \%$ in $\mathrm{w}_{\mathrm{t}, \mathrm{M}}$, and $\approx 5.7 \%$ in $\overline{\mathrm{w}}_{\mathrm{t}, \mathrm{M}, \mathrm{R}}$. Figure 3 and Table 2 indicate that the ratio $R_{\mathrm{N}}=\left(N_{\mathrm{TO}}+N_{\mathrm{A}}\right) / N_{\mathrm{H}}$ for cluster members is larger than or comparable to CS values. The counts, repeated applying a slightly different criterion with the conditions $([\mathrm{OII}]$ AND $\mathrm{H} \beta) \mid([\mathrm{OII}]$ AND [OIII]) | ([OII] AND [NII]) | (H $\beta$ AND [OIII]) | (H $\beta$ AND $\mathrm{H} \alpha) \mid(\mathrm{H} \alpha$ AND $[\mathrm{NII}])$ give consistent results.

Among cluster galaxies we identify 2 Seyfert $1 \mathrm{~s}$ (WINGSJ043838.78-220325.0 and WINGSJ060131.87401646), and one Seyfert 1.9 (WINGSJ034144.52534221.1) One of the four w0 Seyfert galaxies (WINGS J012442.24+085124.4) was modeled with SPECFIT including all relevant components (Marziani et al. 2013). In the other cases (WINGSJ042931.90-613820.0, WINGSJ125732.47-173633.1, 

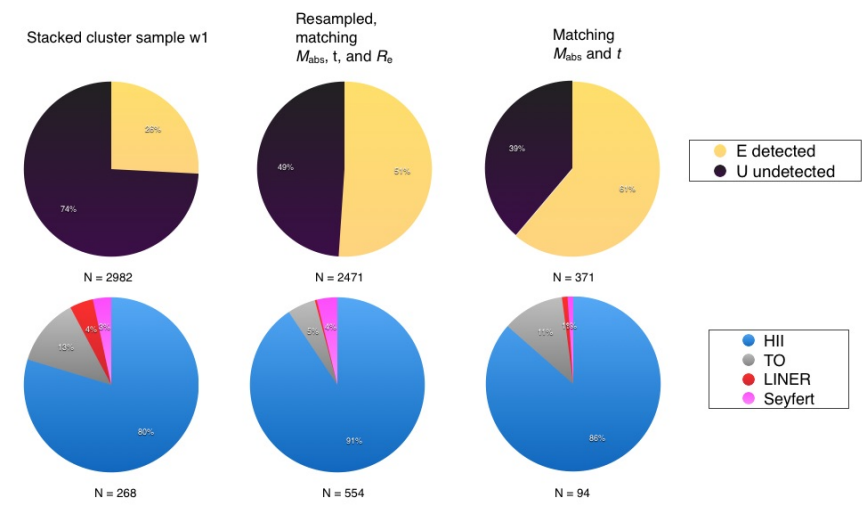

Fig. 3. Top: fractions of quiescent galaxies (black) and ELGs (yellow), and for $\mathrm{w} 1$ cluster galaxies (left), one realization of $\overline{\mathrm{w}}_{\mathrm{t}, \mathrm{M}, \mathrm{R}}$ and $\mathrm{w} 0_{\mathrm{t}, \mathrm{M}}$ (middle and right). Bottom: prevalence of different ELG classes identified in the $\mathrm{DD}_{[\mathrm{NII}]}$ from uncensored diagnostic ratios: HII (blue), transition objects (pale gray), LINERs (red), and Seyferts (magenta), ordered as in the top row.

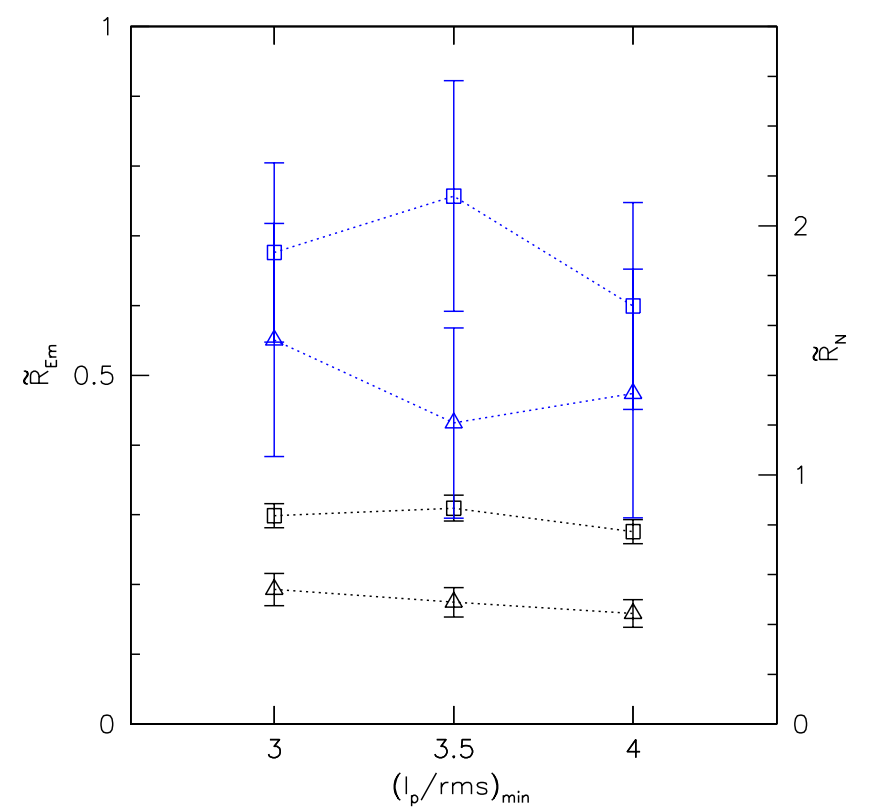

Fig. 4. Effect of assumed minimum $I_{\mathrm{p}} / \mathrm{rms}$ on fraction of ELGs with respect to CSs. The left ordinate shows the ratio $\tilde{R}_{\mathrm{Em}}$ (black lines; squares and triangles indicate normalization by $\overline{\mathrm{w}}_{\mathrm{t}, \mathrm{M}, \mathrm{R}}$ and $\mathrm{w} 0_{\mathrm{t}, \mathrm{M}}$ ) respectively. The right ordinate shows the ratio $\tilde{R}_{\mathrm{N}}$ (blue).

WINGSJ132513.37-313137.7) the broad $\mathrm{H} \beta$ component was not considered in the $\mathrm{H} \beta$ measurements.

We tested the result robustness, that is, that the results are not an artifact of the relatively low minimum $I_{\mathrm{p}} / \mathrm{rms}(=3)$. Figure 4 shows the ratio between $R_{\mathrm{Em}}$ computed for w1 and the CSs $\left(\mathrm{w}_{\mathrm{t}, \mathrm{M}}\right.$ and $\left.\overline{\mathrm{w}}_{\mathrm{t}, \mathrm{M}, \mathrm{R}}\right): \tilde{R}_{\mathrm{Em}}=\left(N_{\mathrm{Em}} / N_{\mathrm{U}}\right)_{1} /\left(N_{\mathrm{Em}} / N_{\mathrm{U}}\right)_{\mathrm{cs}}$, and similarly the ratio: $\tilde{R}_{\mathrm{N}}=\left(N_{\mathrm{N}} / N_{\mathrm{H}}\right)_{1} /\left(N_{\mathrm{N}} / N_{\mathrm{H}}\right)_{\mathrm{cs}}$, where the subscript $\mathrm{cs}$ indicates the number in either one realization of $\mathrm{w}_{\mathrm{t}, \mathrm{M}}$ or $\overline{\mathrm{w}}_{\mathrm{t}, \mathrm{M}, \mathrm{R}}$ for the minimum $I_{\mathrm{p}} / \mathrm{rms}$ equal to $3,3.5$, and 4 (Table 2 and Fig. 4 ; the case of w0 also confirms the stability of the ratio as a function of $I_{\mathrm{p}} / \mathrm{rms}$ ). The ratio $\tilde{R}_{\mathrm{Em}}$ remains $\lesssim 0.3$ for all $I_{\mathrm{p}} / \mathrm{rms}$, indicating that the lower frequency of ELGs in clusters is not dependent on the minimum value of $I_{\mathrm{p}} / \mathrm{rms}$ used as detection criterion. The ratio $\tilde{R}_{\mathrm{N}}$ is $\approx 1.5$ if computed for realizations of the CSs and w1.
The significance of these results was estimated using a bootstrap resampling technique. The upper panel of Fig. 5 compares the median values for the cluster sample to the distribution of $f_{\text {Em }}$ and $R_{\mathrm{N}}$ for $\gtrsim 1000 \mathrm{w} 0_{\mathrm{t}, \mathrm{M}}$ realizations (two leftmost top panels), as well as with 200 realization of the resampled $\overline{\mathrm{w}} 0_{\mathrm{t}, \mathrm{M}, \mathrm{R}}$. The prevalence $f_{\mathrm{Em}}$ remains a factor $\approx 2$ smaller in the cluster sample w1 than in the CSs; $R_{\mathrm{N}}$ is larger by a factor of $\approx 1.5$ (or at least comparable) in w1 with respect to CSs. In the case of $\overline{\mathrm{w}}_{\mathrm{t}, \mathrm{M}, \mathrm{R}}$ the dispersions of the bootstrapped samples are lower because of the significant number of source repetitions that are necessary with this technique (Sect. 2.3).

The lower values of $R_{\mathrm{Em}}$ are in agreement with several previous studies (reviewed in Sect. 5), but the larger (or at least comparable) $R_{\mathrm{N}}$ for cluster galaxies is an intriguing result.

\subsection{Prominence of emission lines: weaker, not just fewer}

Cluster ELGs are not only rarer, they are also weaker. The number of ELGs is approximately halved if a restriction on $W(\mathrm{H} \beta) \geq 3 \AA$ is introduced. For $W(\mathrm{H} \beta) \geq 3 \AA$, the value of $R_{\mathrm{N}}$ becomes $\approx 5 \%$, down from $25 \%$ if no restriction on $\mathrm{EW}$ is applied. This immediately suggests that w1 ELGs are mostly low luminosity. Figure 5 compares the medians of $\log L(\mathrm{H} \beta)$ and $\log W(\mathrm{H} \beta)$ for cluster galaxies and the distributions for the bootstrapped $\mathrm{w}_{\mathrm{t}, \mathrm{M}}$ (left) and $\overline{\mathrm{w}}_{\mathrm{t}, \mathrm{M}, \mathrm{R}}$ (right), including all ELGs regardless of class. The blue lines refer to detections while gray lines alone include upper limits. The $L(\mathrm{H} \beta)$ differences are as large as $0.5 \mathrm{dex}$. The value $W(\mathrm{H} \beta)$ is systematically lower by $\approx 0.5 \mathrm{dex}$ if upper limits are included. Similar considerations apply to the SFR computed from $\mathrm{H} \beta$ (middle panels of Fig. 5), for sources classified as HII, although the difference in median is a factor 1.5-2.5 depending on CS, and to the SFR computed from $\mathrm{H} \alpha$ (middle panels of Fig. 5) for sources classified as HII. We also tested the possibility of a systematic difference for the ratio [OIII] $\lambda 5007 / \mathrm{H} \beta$. The bootstrap analysis again confirms a significant systematic difference with $\Delta \log [\mathrm{OIII}] \lambda 5007 / \mathrm{H} \beta \approx 0.15-0.2$. The distributions of $L(\mathrm{H} \beta)$ and $W(\mathrm{H} \beta)$ for clusters and non-cluster galaxies (including all ELG classes) are found to be statistically different from a confidence level $P \gtrsim 1-0.00005$ by two-sample Wilcoxon generalized tests that include censored data (in this case, upper limits to $L(\mathrm{H} \beta)$ and $W(\mathrm{H} \beta))$, using the SURVIVAL package implemented within IRAF (Feigelson \& Nelson 1985).

Figure 6 shows the distributions of $W(\mathrm{H} \beta)$ for HII and TOs separately. The w1 distribution of $W(\mathrm{H} \beta)$ for HII is apparently bimodal or at least strongly skewed. Clusters galaxies show a tail of low-W HII sources that may contribute to the highly significant difference in average equivalent width revealed by the boostrap analysis (Fig. 5). Instead, TOs show a single peaked distribution that is clearly shifted toward higher values for w0 and two CSs (apart from fluctuations due to small numbers). A Peto-Prentice generalized Wilcoxon test has been applied for comparing $\mathrm{w} 1$ and bootstrapped $\mathrm{w}_{\mathrm{t}, \mathrm{M}}$ and $\overline{\mathrm{w}}_{\mathrm{t}, \mathrm{M}, \mathrm{R}}$, now separating HII, TOs, LINERs, and Seyferts, including censored data. The differences in $\mathrm{H} \beta$ equivalent width are highly significant for TOs and HII: the Peto-Prentice test statistic is $>3$ (TOs) and $>5$ (HII) for $\approx 200$ bootstrapped samples, implying $P \lesssim 0.025$ and $\lesssim 0.00005$ that w1 and control samples are randomly drawn from the same population. While $\mathrm{H} \beta$ luminosity is significantly higher for HII in the CSs, it is not so for TOs if $\mathrm{w}_{\mathrm{t}, \mathrm{M}}$ is compared to $\mathrm{w} 1$; but the differences become highly significant for all bootstrap realizations in $\overline{\mathrm{w}}_{\mathrm{t}, \mathrm{M}, \mathrm{R}}$ (both $\mathrm{H} \alpha$ and $\mathrm{H} \beta$ ). The suggested implication is that TOs are characterized by a deficit of luminosity 

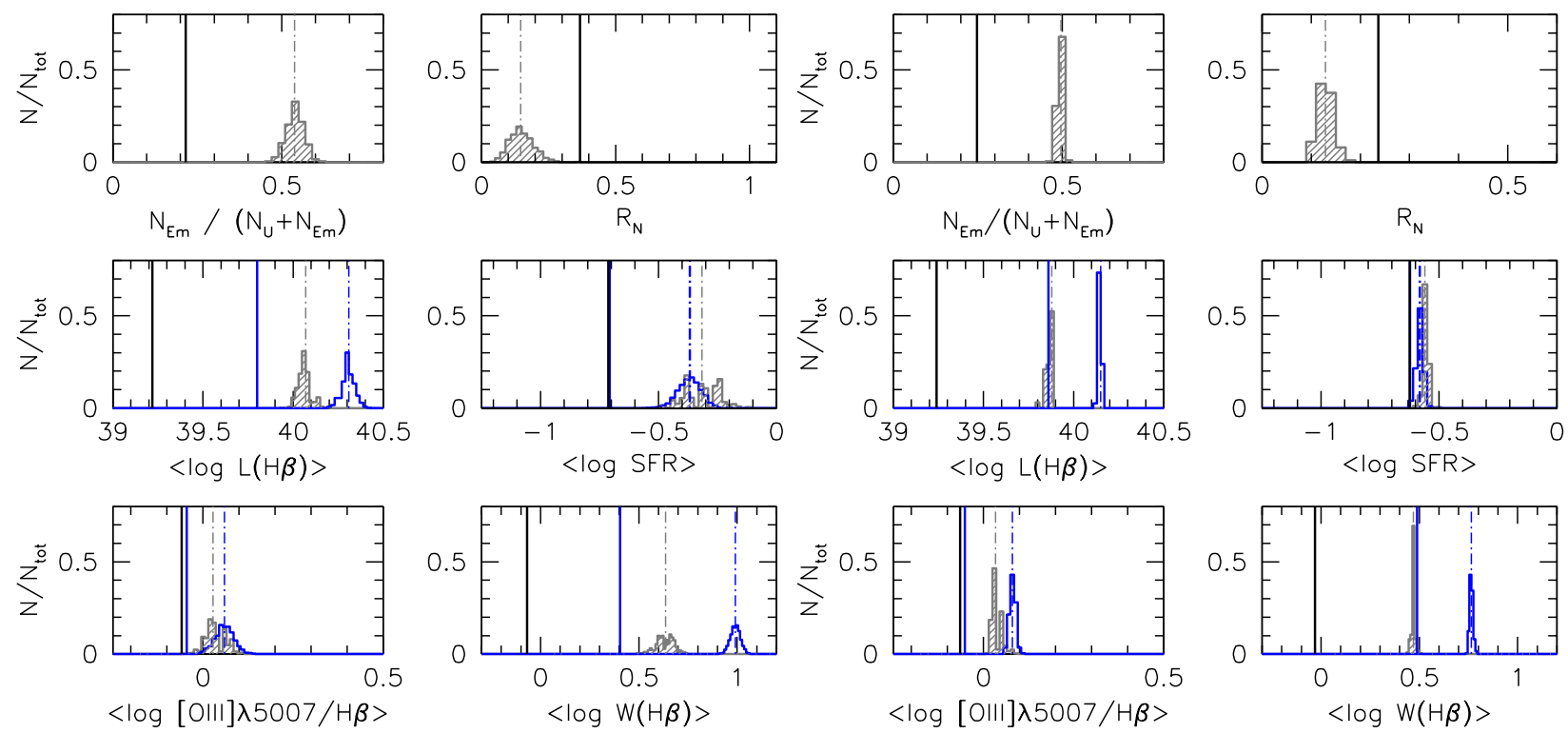

Fig. 5. Results of bootstrap simulations. The $\mathrm{w} 1$ sample is compared with 1000 virtual $\mathrm{w}_{\mathrm{t}, \mathrm{M}} \mathrm{CSs}$ of $\approx 350$ sources (six leftmost panels), and with 200 virtual $\overline{\mathrm{w}}_{\mathrm{t}, \mathrm{M}, \mathrm{R}}$ (six rightmost panels). The panels show median values measured for the cluster members (thick solid lines) along with the distributions of the bootstrapped sample $\mathrm{wO}_{\mathrm{t}, \mathrm{M}}$ medians $(l e f t)$ and $\overline{\mathrm{w}}_{\mathrm{t}, \mathrm{M}, \mathrm{R}}$ (right) and their medians (dot-dashed lines). Top: ratio $R_{\mathrm{Em}}(l e f t)$ and ratio $R_{\mathrm{N}}$ (from $\mathrm{DD}_{[\mathrm{NII}]}$; right) for the revised classification (gray). The middle panels show the luminosity of $\mathrm{H} \beta$ in erg $\mathrm{s}^{-1}$ (left), and the SFR in $M_{\odot} \mathrm{yr}^{-1}$ (only for HII sources; right). Blue histograms and lines refer to average values of detections only, gray histogram and lines show medians and include upper limits. Bottom panel: distributions for [OIII] $\lambda 5007 / \mathrm{H} \beta($ left $)$ and $W(\mathrm{H} \beta)($ right $)$, with the same meaning of color coding.

per unit mass, rather than a simple general luminosity deficit (as further discussed in Sect. 5.1). The Peto-Prentice test indicates lower [OIII] $\lambda 5007 / \mathrm{H} \beta$ (at a $2 \sigma$ confidence level) for HII and TOs in more than $90 \%$ of the both $\mathrm{w}_{\mathrm{t}, \mathrm{M}}$ and $\overline{\mathrm{w}}_{\mathrm{t}, \mathrm{M}, \mathrm{R}}$ realizations. Differences between $\mathrm{w} 1$ and $\overline{\mathrm{w}}_{\mathrm{t}, \mathrm{M}, \mathrm{R}}$ in $\mathrm{H} \beta \mathrm{EW}$ and luminosity are also significant for the Seyfert class. If a restriction is carried out to uncensored line ratios including the $\mathrm{H} \alpha$ range, differences in $L(\mathrm{H} \beta)$ and $W(\mathrm{H} \beta)$ become not significant for HII and TOs, alike; this implies, however, a restriction to the stronger emitters with $W(\mathrm{H} \beta) \gtrsim$ a few $\AA$.

\subsection{Diagnostic diagrams: a population of low ionization AGNs and TOs in cluster}

The diagnostic diagrams $\mathrm{DD}_{[\mathrm{NII}]}, \mathrm{DD}_{[\mathrm{OII}]}$, [OIII $] \lambda 5007 / \mathrm{H} \beta$ versus $[\mathrm{OI}] \lambda 6300 / \mathrm{H} \beta$ and $[\mathrm{SII}] \lambda \lambda 6716,6731 / \mathrm{H} \alpha$ (hereafter $\mathrm{DD}_{[\mathrm{OI}]}$ and $\mathrm{DD}_{[\mathrm{SII}]}$, respectively) are shown in Figs. $7-10 ; S / N_{\min }=3$ is assumed. The four panels in each figure show the w1, the w0, one realization of $\mathrm{w}_{\mathrm{t}, \mathrm{M}}$ and of $\overline{\mathrm{w}}_{\mathrm{t}, \mathrm{M}, \mathrm{R}}$. The diagnostic diagrams that include censored data confirm the trends derived from the diagrams only involving detections.

The information provided by each individual diagram is largely independent and subject to different biases $\left(\mathrm{DD}_{[\mathrm{OII}]}\right.$ : internal reddening; $\mathrm{DD}_{[\mathrm{OI}]}$ and $\mathrm{DD}_{[\mathrm{SII}]}$ : very faint lines and predominance of upper limits). For instance, the rarity of LINERs and Seyferts in the $\mathrm{DD}_{[\mathrm{OII}]}$ diagram (Fig. 8) is most likely due to a bias, that is, to a systematic underestimate of $[\mathrm{OII}] \lambda 3727 / \mathrm{H} \beta$, since no internal reddening correction was applied. Sources in the LINER and Seyfert area of $\mathrm{DD}_{[\mathrm{OII}]}$ should be properly classified, but there could be a significant fraction of LINERs and Seyferts that are improperly classified as HII because [OII] $\lambda 3727$ is underestimated. In the on-line WINGS database described in Sect. 4.1, we report four source classifications from the different diagrams (record headings are listed in Table B.1).

Revised classifications following the approach of Appendix A.1 are only assigned to sources that enter into the
$\mathrm{DD}_{[\mathrm{NII}]}$ and $\mathrm{DD}_{[\mathrm{OII}]}$. The revision affects individual source classification but does not change any qualitative statistical result reported in the paper. The number of TOs is significantly reduced after revision if censored data are considered but the relation between w1 and controls is still highly significant because both cluster and control prevalences are reduced by similar amounts. If no censored line ratios are considered, the count change is more modest, involving only $\sim 10 \%$ of sources that are mainly reclassified as HII (Table 2).

Figure 7 emphasizes the abundance of TOs and LINERs in the cluster sample. We stress again that the TO and the LINER populations of cluster members almost completely disappear if samples are restricted to large Balmer line EWs $(\gtrsim 3 \AA)$ as can be deduced from the number counts of Table 2. TOs and LINERs are populations of predominantly weak emitters.

A large percentage of TOs classified with $\mathrm{DD}_{[\mathrm{NII}]}$ are also present in $\mathrm{DD}_{[\mathrm{OI}]}$ and $\mathrm{DD}_{[\mathrm{SII}]}$ and are classified as LINERs (90\%), adding further support to the hypothesis that TOs are genuinely different from pure HII regions, at least in the cluster environment. LINERs are apparently more frequent than Seyferts in $w 1$, if we trust low equivalent width sources close to the detection limit: $\mathrm{w} 0_{\mathrm{t}, \mathrm{M}}$ most frequently does not collect any LINER at all; the average ratio $N_{L} / N_{S} \approx 0.35$ for 1000 realization of $\mathrm{w} 0_{\mathrm{t}, \mathrm{M}}$, and $\approx 1$ for $\mathrm{w} 1$.

\subsection{The diagram of Cid Fernandes et al. (2010): an excess of retired sources}

The emission line weakness of cluster galaxies is made even more explicit in the plane $W(\mathrm{H} \alpha)$ vs. [NII] $\lambda 6583 / \mathrm{H} \alpha$ (Fig. 11; meaning of symbols and arrangement of panels is the same as in the previous figures). Sources are separated into strong AGNs, weak AGNs, HII, and retired galaxies. The latter class includes sources for which $W(\mathrm{H} \alpha) \leq 2.5 \AA$ (Cid Fernandes et al. 2010) i.e., old galaxies with weak emission lines whose spectra are similar to those of LINERs (Stasińska et al. 2008, 2015). 
HII
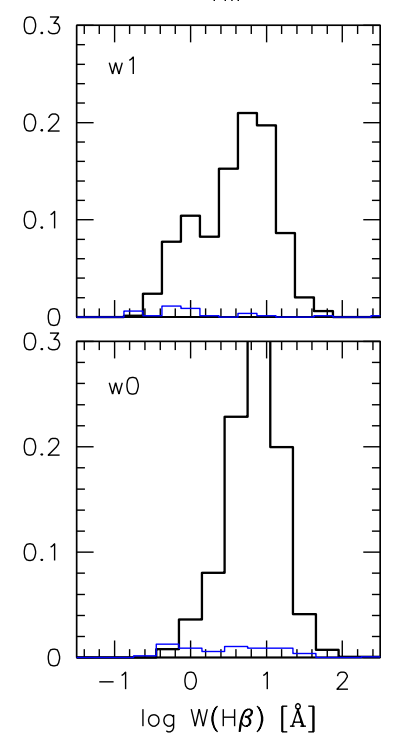

HII

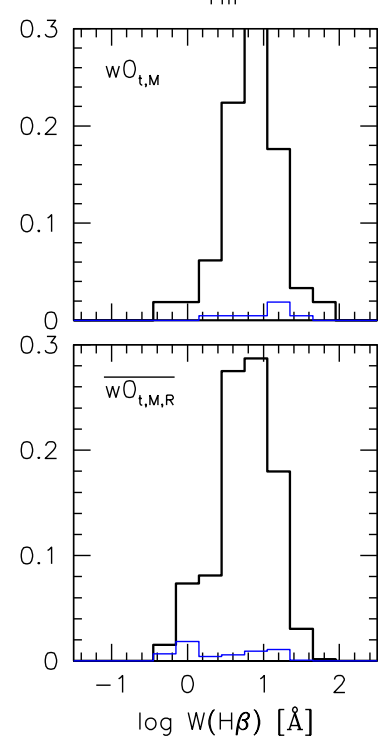

TO

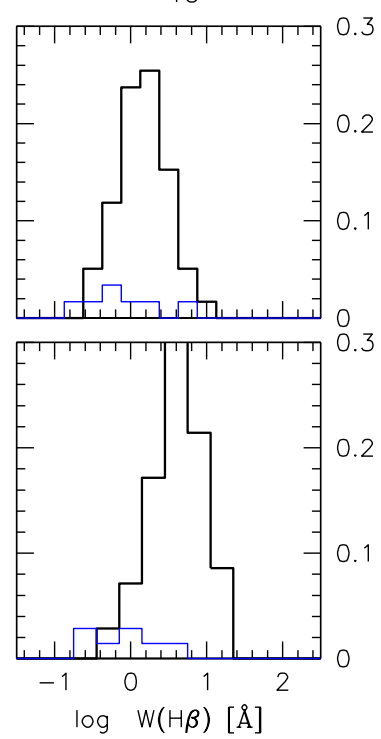

TO

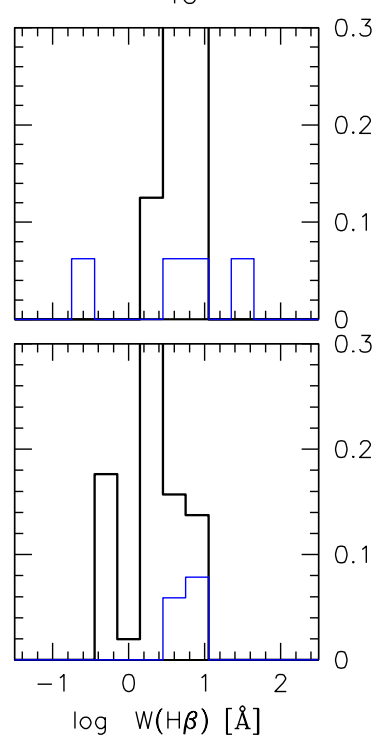

Fig. 6. Distribution of $W(\mathrm{H} \beta$ ) (in $\AA$ ) for sources classified as HII (left), and TO (right), for $\mathrm{w} 1, \mathrm{w} 0$, one realization of $\mathrm{w}_{\mathrm{t}, \mathrm{M}}$ and $\overline{\mathrm{w}}_{\mathrm{t}, \mathrm{M}, \mathrm{R}}$ (from top to bottom). The black histograms trace the distributions of detections, and the thin blue histograms trace the distributions of upper limits.

Especially striking is the systematic difference in $W(\mathrm{H} \alpha)$ for TO between cluster (left) and non-cluster galaxies: $W(\mathrm{H} \alpha)$ in TOs appears weaker by a factor of 10 . In addition, cluster members tend, much more frequently, to occupy the area of retired galaxies, which is scarcely populated even in the full sample of non-cluster galaxies. The CSs on the right confirm these trends, even if, also owing to the lower $R_{\mathrm{N}}$ ratio, relatively few objects populate the area of the diagram with $\log [\mathrm{NII}] \lambda 6583 / \mathrm{H} \alpha \gtrsim-0.3$ in the non-member samples.

A first possibility in the interpretation of TOs is that we are simply witnessing the same scaled-down phenomenon as in non-cluster galaxies. However, the diagram $W(\mathrm{H} \alpha)$ versus $[\mathrm{NII}] \lambda 6583 / \mathrm{H} \alpha$ argues against the suggestions that TOs in clusters are due to a mixture of gas photoionized by a hot stars and an AGN: in this case, no decrease in line EW is expected.

\section{Discussion}

In the following, we discuss the nature of the non-HII emitters (Sects. 5.1 and 5.2) without entering the details of individual cluster and galaxy properties. We compare the present work and other surveys of ELGs for clusters (Sect. 5.3), and works for different surroundings (compact groups and isolated galaxies, Sect. 5.4).

\subsection{On the nature of non-HII LL ELGs in cluster. Almost quiescent line emitters}

Systematically weaker lines account for the placement of most ELGs in clusters among retired galaxies and for the almost vertical displacement in the diagram of Fig. 11 (cf. Pimbblet et al. 2013). A change in the amount of ionized gas at a given level of stellar continuum emission is implied by the definition of EW. We also show $L(\mathrm{H} \beta)$ versus stellar mass $M_{\star}$ in Fig. 12 (a similar trend hold for $\mathrm{H} \alpha$, and is not shown), where $M_{\star}$ has been collected from Fritz et al. (2011), and the mass value is their Mass 1, i.e., the sum of the masses of all stars ever formed and of the remaining gaseous component. An intriguing difference between the w1 and CSs is related to the $L(\mathrm{H} \alpha)$ distribution of HII sources; a fraction of sources shows comparable values in w1 and CSs but w1 also shows a significant low- $L$ tail that is probably associated with gas depletion. This trend is present over the whole $M_{\star}$ range considered in Fig. 12, and is probably reflected in the $W(\mathrm{H} \beta)$ distribution of HII sources as well (Fig. 6). The TO $L(\mathrm{H} \alpha)$ distribution is affected by some very massive hosts at $\log M_{\star} \gtrsim 11\left[M_{\odot}\right]$, which are poorly represented in the CSs. If the mass range is restricted to $9 \lesssim \log M_{\star} \gtrsim 11$, then the $L(\mathrm{H} \alpha)$ is systematically lower than the CSs, confirming a lower amount of emitting gas per unit mass, as emphasized by the equivalent width trends. Because of their low equivalent width the wide majority of TOs belong to the $e(c)$ (moderate to weak emission lines) and $k$ (resembling $\mathrm{K}$ stars with no emission lines) in the spectral classification devised by Fritz et al. (2014). Retired ELGs are not associated with jellyfish galaxies (Poggianti et al. 2016); jellyfish galaxies belonging to the w1 sample show prominent emission lines, often with HII spectrum.

The color magnitude diagram (Fig. 13) $B-V$ vs. $M_{V}$ for the w1 sample shows that the TOs are mainly located along the quiescent population of early-type galaxies (where LINERs are also found) or, to a lesser extent in the so-called green valley where X and IR selected AGN are usually found (e.g., Fang et al. 2012, and references therein).

\subsection{Shocks induced by ram stripping?}

An outstanding result of this investigation is the placement of most cluster TO and LINER sources in the area of retired galaxies of Fig. 11. From Fig. 11 we see that this is not the case either for sample w0 nor for the CSs. The non-cluster LINER and TO populations are preferentially placed in the strong and weak AGN region of $\log W(\mathrm{H} \alpha) \gtrsim 0.4$ and $\lesssim 0.4$, respectively. In the AGN interpretation, this may mean that TOs and LINERs in clusters are fainter versions of their field counterpart. However, the systematic differences in equivalent width and luminosity and the relatively infrequent detection in X-rays leave open the possibility that cluster LINER and TOs may be due to a different physical mechanism. Recent works suggest that X-ray-detected AGNs are under-represented in the cluster central regions (e.g., Koulouridis \& Plionis 2010; Ehlert et al. 2014), even if cluster LINERs are not recognized as X-ray emitters, as the X-ray 
P. Marziani et al.: Active galactic nuclei in WINGS
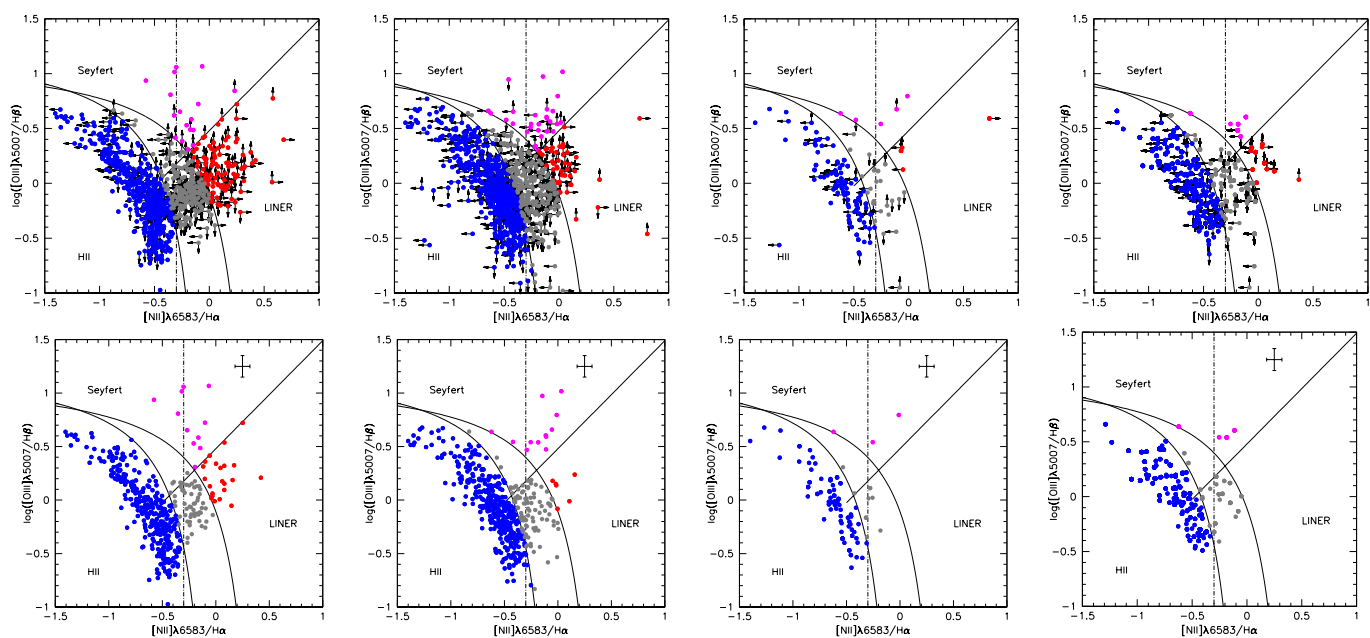

Fig. 7. Diagnostic diagrams of [OIII] $\lambda 5007 / \mathrm{H} \beta$ vs. [NII] $65583 / \mathrm{H} \alpha$. Leftmost panel: DD for galaxies that are cluster members; second panel from right: same, for $\mathrm{w} 0$. The third and fourth panels from right show one realization of $\mathrm{w}_{\mathrm{t}, \mathrm{M}}$ and of $\overline{\mathrm{w}}_{\mathrm{t}, \mathrm{M}, \mathrm{R}}$. Blue, red, and magenta colors identify HII, LINERs, and Seyferts, respectively. Gray data points identify transition objects. Arrows indicate data points for which upper and/or lower limits to the diagnostic emission line ratios are considered. The bottom rows show panel for detections ordered in the same sequence. Median errors at a $1 \sigma$ confidence level are shown in the upper right corner of the diagrams with detections only.
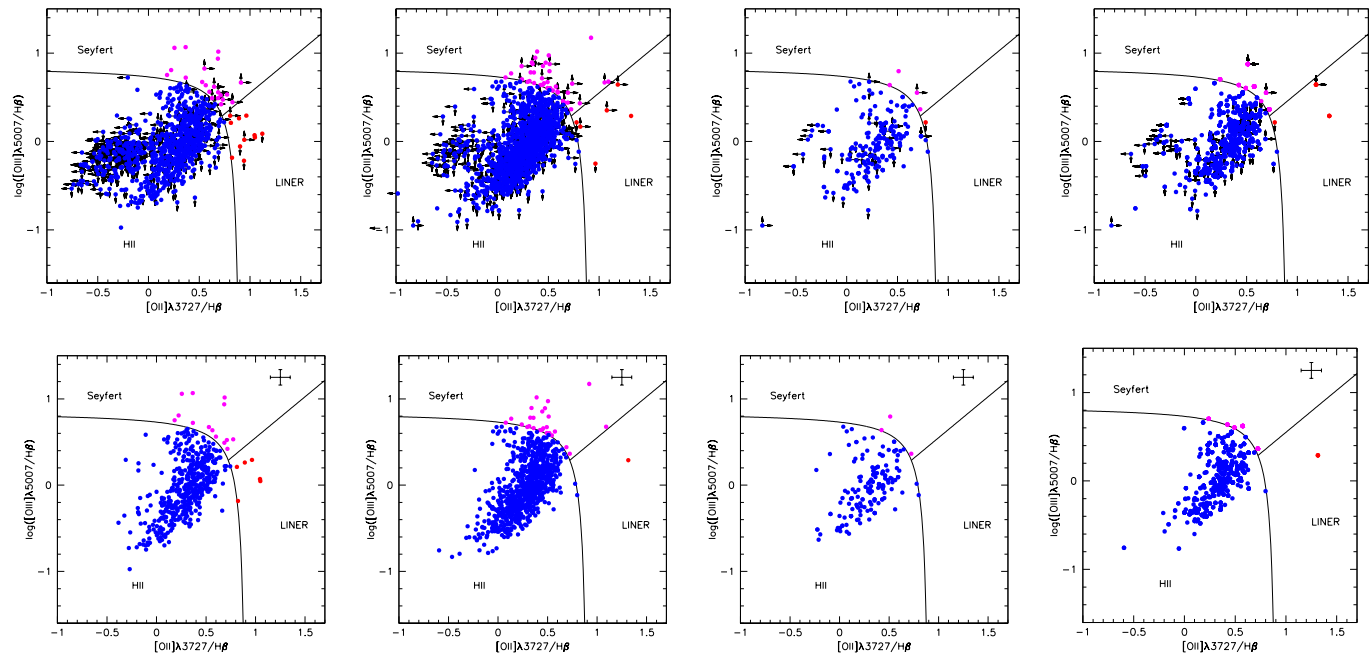

Fig. 8. Diagnostic diagram [OIII] $\lambda 5007 / \mathrm{H} \beta$ vs. [OII] $] \lambda 3727 / \mathrm{H} \beta$. Meaning of panel and symbols and disposition of panels is the same as Fig. 7.
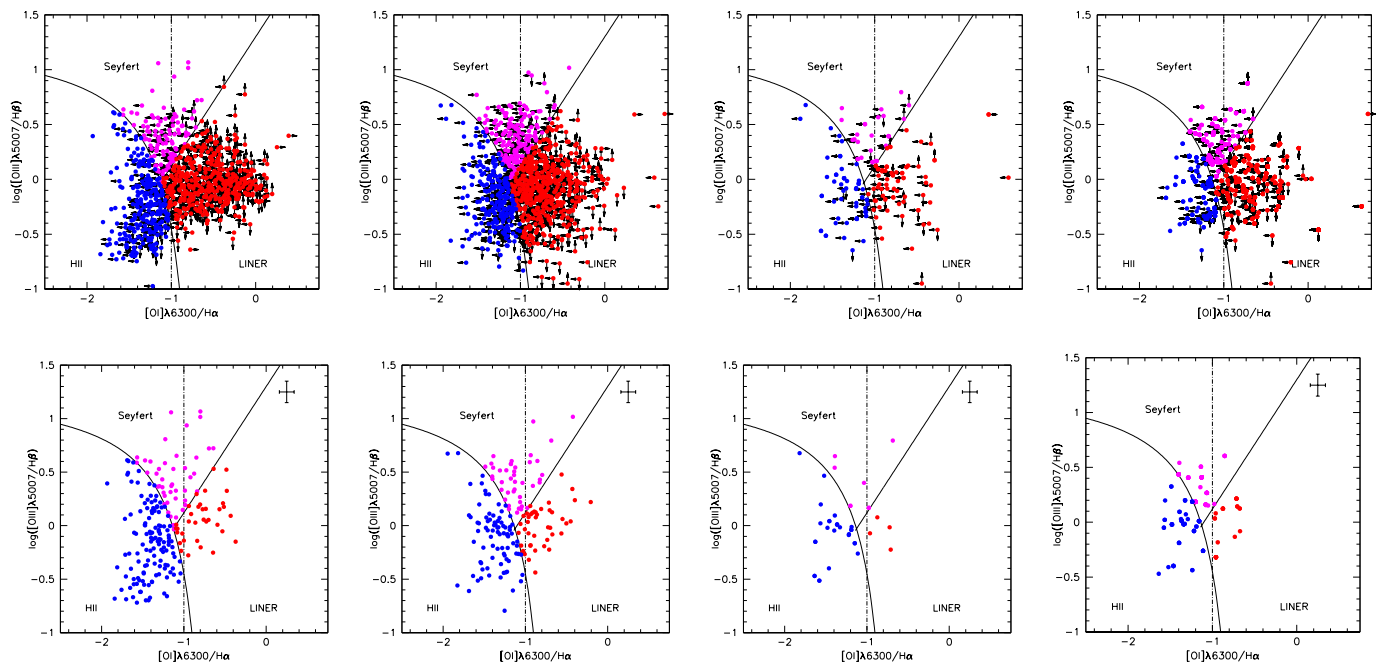

Fig. 9. [OIII] $\lambda 5007 / \mathrm{H} \beta$ vs. [OI] $\lambda 6300 / \mathrm{H} \alpha$ diagnostic diagrams. Meaning of panel and symbols and disposition of panels is the same as Fig. 7 

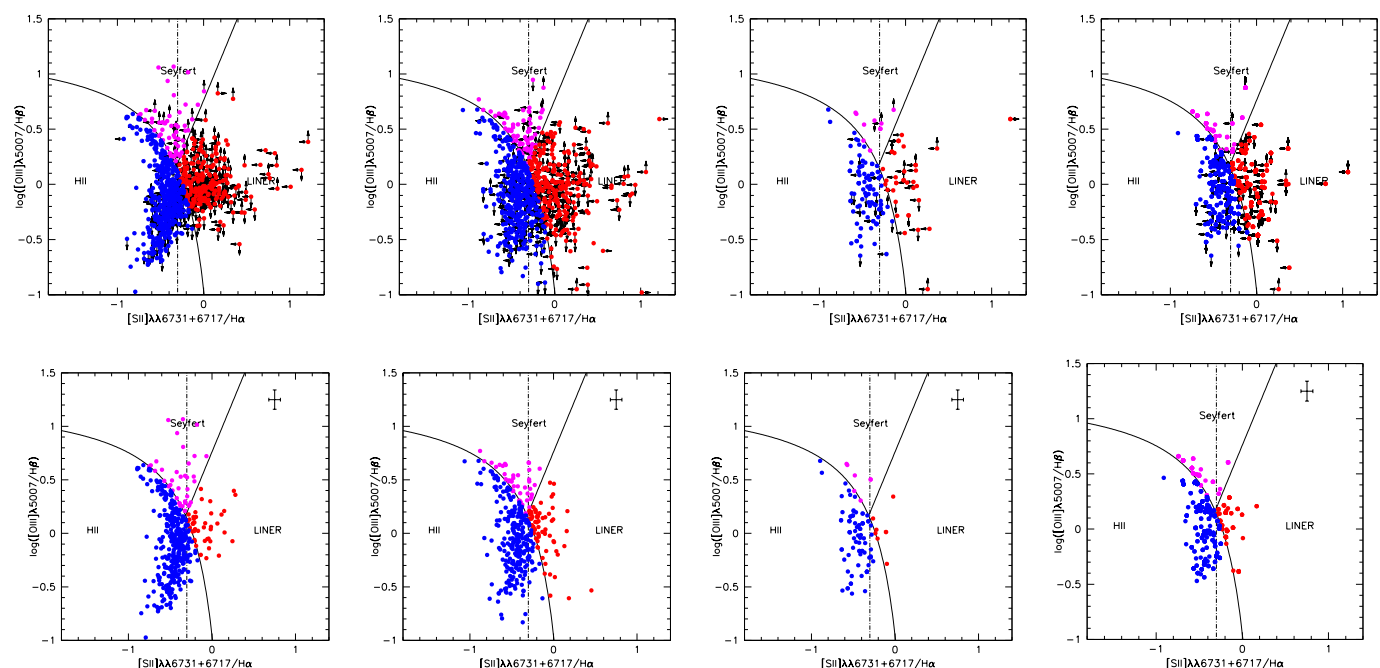

Fig. 10. [OIII] $\lambda 5007 / \mathrm{H} \beta$ vs. [SII] $\lambda \lambda 6716,6731 / \mathrm{H} \alpha$ diagnostic diagrams. Meaning of panel and symbols and disposition of panels is the same as Fig. 7 .
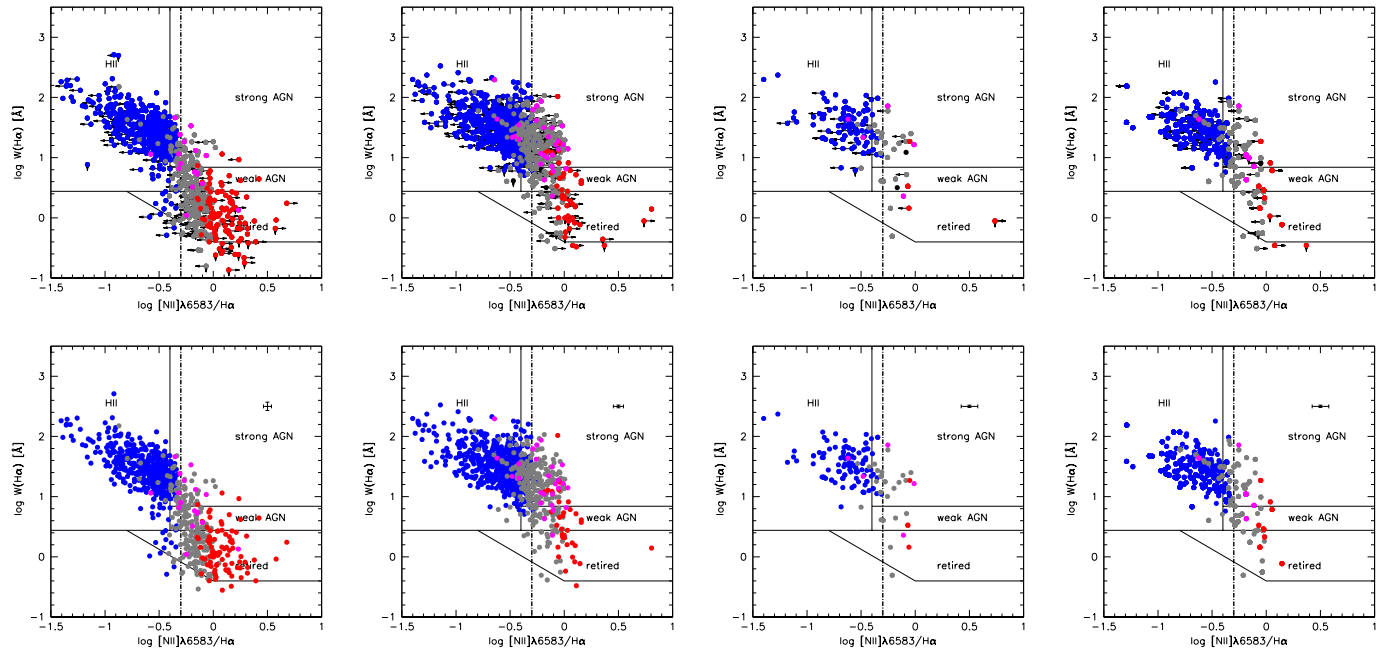

Fig. 11. Equivalent width of $\mathrm{H} \alpha W(\mathrm{H} \alpha)$ vs. diagnostic ratio [NII] $\lambda 6583 / \mathrm{H} \alpha$. Upper panels: sources with detections and upper and lower limits. Bottom panels: sources with detection only. Leftmost panel are for sources belonging to clusters, panels on the right are for control sources: w0 (second from left) and one realization of $\mathrm{w}_{\mathrm{t}, \mathrm{M}}$ and of $\overline{\mathrm{w}}_{\mathrm{t}, \mathrm{M}, \mathrm{R}}$. Dividing lines are drawn according to Cid Fernandes et al. (2010), separating HII, strong AGNs, weak AGNs, and retired galaxies. The dot-dashed line indicates a limiting [NII] $65583 / \mathrm{H} \alpha$ ratio for extragalactic nuclear HII region. Color coding identifies HII, TO, LINERs, and Seyfert as in the previous figures. Black data points identify ELGs with no entry in DD ${ }_{[N I I]}$.

detection of individual cluster galaxies is hampered by flux and resolving power limits, and Compton-thick LINERs may be undetected in the soft X-ray domain (González-Martín et al. 2015).

Observationally, line emission from shock-heated gas with a LINER-like spectrum has been detected in a variety of situations associated with strongly interacting systems (such as for example Kar 23) with molecular cloud collisions (e.g., Marziani et al. 1994, 2001; Appleton et al. 2006; Ogle et al. 2007; Monreal-Ibero et al. 2010; Merluzzi et al. 2013). However, the shock phenomenology is not limited to interacting galaxies. A warm molecular hydrogen tail due to ram pressure stripping has been detected in a cluster galaxy, ESO 137-001 (Sivanandam et al. 2010). Sivanandam et al. (2014) provide imaging in both $\mathrm{H} \alpha$ and $\mathrm{H}_{2}$ line at 17. $\mu \mathrm{m}\left(\mathrm{H}_{2} 0-0 \mathrm{~S}(1)\right.$ transition) for four cluster galaxies, showing a close association between $\mathrm{H} \alpha$ emission and part of the $\mathrm{H}_{2}$ emission owing to molecular shocks. An especially interesting case in this respect is the one of NGC 4522, where the $\mathrm{H} \alpha$ and $\mathrm{H}_{2}$ emission extend across the inner galaxy disk.

The mass of ionized gas needed to account for the observed $\mathrm{H} \alpha$ luminosity is $M_{\mathrm{H}^{+}}=L(\mathrm{H} \alpha) m_{\mathrm{p}} / n \alpha_{\mathrm{H} \alpha} h v_{\mathrm{H} \alpha}$, where $m_{\mathrm{p}}$ is the proton mass, $n$ the number density, $\alpha_{\mathrm{H} \alpha}$ the effective recombination coefficient for $\mathrm{H} \alpha$ (Osterbrock \& Ferland 2006), $h$ the Planck constant, and $v_{\mathrm{H} \alpha}$ the frequency of the $\mathrm{H} \alpha$ photons. For the conditions appropriate in the ISM medium $M_{\mathrm{H}^{+}} \approx$ $2 \times 10^{5} L(\mathrm{H} \alpha)_{39} n_{10}^{-1} M_{\odot}$ (with $L(\mathrm{H} \alpha)$ in units of $10^{39} \mathrm{erg} \mathrm{s}^{-1}$, and $n$ of $10 \mathrm{~cm}^{-3}$ ), which is a modest amount even for the gasdeficient cluster galaxies. Energetically, shock emission from $n=1 \mathrm{~cm}^{-3}$ gas would imply a covering factor that can be well $f_{\mathrm{c}} \sim L(\mathrm{H} \alpha) / f_{\Sigma} \pi R_{\mathrm{e}}^{2} \sim 10^{-1} \ll 1$ (where $f_{\Sigma}$ is the surface emissivity $\sim 10^{-1} \mathrm{erg} \mathrm{s}^{-1} \mathrm{~cm}^{-2}$; Allen et al. 2008).

Shock-heating models appropriate to HI gas account for the observed emission line ratios of TOs and LINERs. Observed emission line ratios, assuming that all line luminosity is due to shocks, can be explained by moderate velocity shocks with 

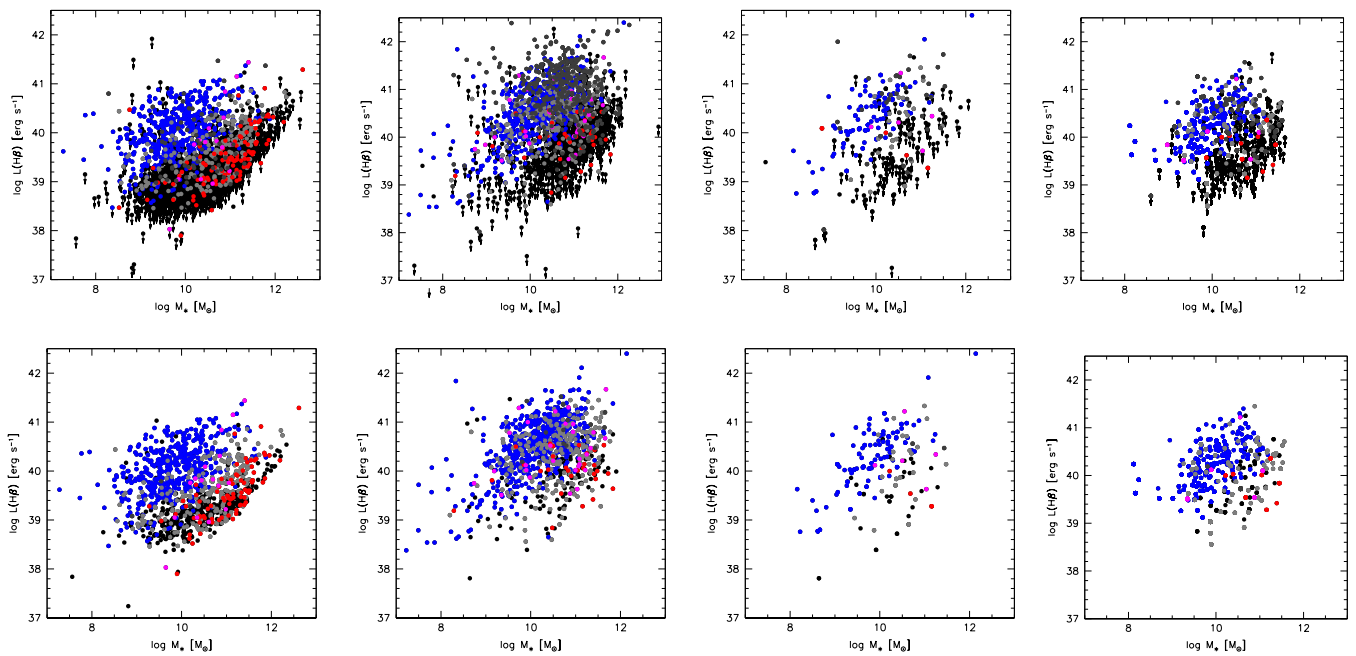

Fig. 12. Logarithm of $\mathrm{H} \beta$ luminosity in erg $\mathrm{s}^{-1}$ vs. log of stellar mass in solar units for cluster members and non-members (middle and right panels) in the following order from left to right: $\mathrm{w} 1, \mathrm{w} 0$, one realization of $\mathrm{w}_{\mathrm{t}, \mathrm{M}}$, and one realization of $\overline{\mathrm{w}}_{\mathrm{t}, \mathrm{M}, \mathrm{R}}$. Upper panels include upper limits and detections, and lower panels are for detections only. Sources of different classes are identified by the same color coding as the previous figures.

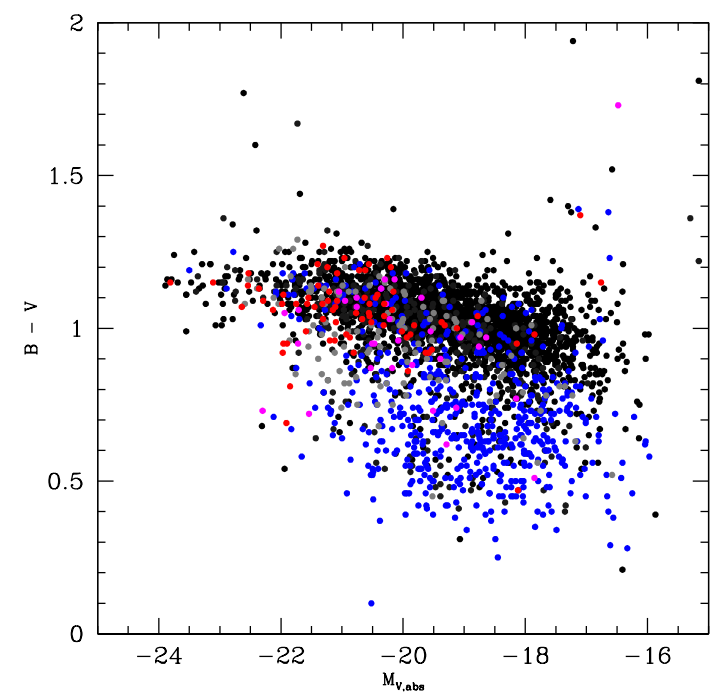

Fig. 13. Color magnitude diagram $B-V$ within a 5 kpc aperture vs. $M_{V}$ for the galaxies of the cluster sample w1. Color coding is the same as in the previous figure.

precursors (i.e., with ionization in advance of the shock front provided by gas heated in the post-shock zone). The extension to the right of data points that enter into the region of LINERs in $\mathrm{DD}_{[\mathrm{NII}}$ (also known as the right wing of the seagull) can be explained as in Fig. 31 of Allen et al. (2008); that is as due to shocks with precursor and shock velocity $\$ 500 \mathrm{~km} \mathrm{~s}^{-1}$. A grid of models with the same limits in shock velocity accounts in part for the distribution of data points in $\mathrm{DD}_{[\mathrm{OI}]}$, although the observed $[\mathrm{OIII}] \lambda 5007 / \mathrm{H} \beta$ ratio is lower than model prediction for the case of shock plus precursor. At face value the Allen et al. (2008) grid computations would suggest shocks without a precursor and a large magnetic parameter that would lower shock compression and hence lead to lower post-shock temperatures. However, we stress that inferences from $\mathrm{DD}_{[\mathrm{OI}]}$ are especially speculative since most measures are upper limits.

Evidence exists that part of the molecular gas content can be stripped by ram pressure, possibly in a sort of progressive ablation at the rims of the molecular gas disk (Rich et al. 2011; Sivanandam et al. 2014). However, molecular gas content is apparently less affected in cluster environments, or, at least, stripped less efficiently than atomic gas (Boselli et al. 2014), probably because of the larger extent of the HI disk, and of the different hydrodynamical effects of the ICM on atomic and molecular gas. The evaporation timescale $t_{\text {evap }}$ depends on cloud size, density, and ICM temperature: $t_{\text {evap }} \propto n_{\mathrm{c}} r_{\mathrm{c}}^{2} T_{\text {ICM }}^{-2.5}$ (Cowie \& McKee 1977) and $t_{\text {evap }}$ is much longer for molecular clouds $\left(n_{\mathrm{c}} \sim 10^{6} \mathrm{~cm}^{-3}\right)$ than for atomic clouds $\left(n_{\mathrm{c}} \sim 10^{1-2} \mathrm{~cm}^{-3}\right)$ of the same size. In the molecular case, $t_{\text {evap }}$ can easily exceed the dynamical timescales for cluster galaxies and may even exceed the Hubble time. Thus the molecular gas could represent a permanent, or at least a long-lived reservoir of gas in cluster galaxies, leading to non-negligible star formation and to a mixed starburst/shock or to a shock phenomenology in a large percentage of cluster galaxies. Mechanical heating and induced star formation may replenish cluster galaxies of atomic gas from molecular clouds (e.g., Hidaka \& Sofue 2002).

\subsection{Comparison with previous works for cluster galaxies}

A quantitative comparison with previous studies is not easy because of differences in luminosity, morphology, and redshift in the galaxy samples and because of data heterogeneity, that is, differences in emission line detection limits associated with $\mathrm{S} / \mathrm{N}$ and spectral resolution. We limit ourselves to elementary considerations on $R_{\mathrm{Em}}, R_{\mathrm{N}}$, and type $1 \mathrm{AGNs}$, and to systematic differences in line luminosity.

Prevalence of ELGs. The notion that ELGs in clusters are significantly less common than in the field and other environments has been consolidated by studies spanning more than 30 years (e.g., Gisler 1978; Balick \& Heckman 1982; Dressler et al. 1985). This basic result has remained mostly unchallenged until now (see however Biviano et al. 1997 for a different view), and is confirmed by the present works for the X-ray bright clusters of the WINGS - SPE survey. There is an overall consistency concerning qualitative trends with the Hwang et al. (2012) results for clusters regarding the low prevalence of ELGs in clusters. 
AGN+TO fraction $f_{\mathrm{N}}$. The prevalence of non-HII ELGs, $f_{\mathrm{N}}$ (not normalized by $N_{\mathrm{H}}$ i.e., $f_{\mathrm{N}}=f_{\mathrm{AGN}}$, where $f_{\mathrm{N}}$ is as defined by Hwang et al. 2012) in the WINGS cluster sample is only slightly lower than in w0 and CSs. The data of Table 2 show that, after redistributing unclassified ELGs, $f_{\mathrm{N}} \approx 5 \%$ for $\mathrm{w} 1$ is comparable to the prevalences $\approx 4 \%$ and $6 \%$ for $\mathrm{w}_{\mathrm{t}, \mathrm{M}}$ and $\overline{\mathrm{w}}_{\mathrm{t}, \mathrm{M}, \mathrm{R}}$ respectively; these prevalences become $\approx 10 \%$ if identifications based on censored ratios are included. The WINGS values that include censored data are in agreement with recent $f_{\mathrm{N}}$ determinations; if the $\mathrm{C} 1$ and $\mathrm{C} 2$ samples of Hwang et al. (2012) are joined, their $f_{\mathrm{AGN}}$ is $\approx 15 \%$. If a restriction to strong emission lines is introduced $\left(W_{\min }=3 \AA\right.$ ), the non-HII prevalence in clusters is lower than in the $\overline{\mathrm{w}}_{\mathrm{t}, \mathrm{M}, \mathrm{R}}$ by a factor $\approx 1.5$ (2.5 if censored data are included), in qualitative agreement with older studies that found a lower prevalence in clusters with respect to less dense environments. The non-HII sources we detect as TOs are mainly of low equivalent width, to the point of being located in the retired galaxy region of Fig. 11, and their contribution might have been missed in past surveys.

$R_{\mathrm{N}}$. The $R_{\mathrm{N}}$ ratio, like $f_{\mathrm{N}}$, can be measured with some precision because it involves a large number of galaxies. The $R_{\mathrm{N}}$ values found by the present work and by Hwang et al. (2012) are different but consistently high $(\approx 0.26$ versus 0.86 , again joining samples $\mathrm{C} 1$ and $\mathrm{C} 2$ of Hwang et al. 2012). The values probably reflect differences in the application of diagnostic diagrams and in cluster-centric distance coverage.

True (or pure) AGNs. The prevalence of AGNs (true AGNs excluding TOs) in our sample is not statistically different from those in the CSs: $\approx 1 \%$ for uncensored diagnostic ratios (Table 2) and $\approx 3 \%$ if censored data are included. This value is consistent with the prevalence found for the $\mathrm{C} 1+\mathrm{C} 2$ sample of the recent Hwang et al. (2012) work.

Type 1.0 AGN. We found two type 1 sources (WINGSJ043838.78-220325.0 and WINGSJ060131.87401646), i.e., one-half of the detected type 2 AGN, as expected for our sample size on the basis of orientation-based AGN unification schemes (Antonucci 1993). If the host galaxy is gas rich, even slight tidal disturbances, i.e., disturbances not as remotely dramatic as mergers like harassment (Moore et al. 1996; Hwang et al. 2012) can trigger nuclear activity. Star formation and type 2 nuclear activity may be concomitant with type 2 activity delayed with respect to star formation but observed as contemporary in a large number of systems, which may account for at least some TOs. However, type 1 activity may be triggered by interaction but may be associated with a significant delay (e.g., Krongold et al. 2003; Koulouridis et al. 2013; Villarroel \& Korn 2014) so that the close environment may not appear different from the environments of non-active galaxies. In this case, gaining evidence of past interactions requires a much more thorough analysis of the type 1 host morphology and environment. On the other hand, active galaxies in clusters are presumed to be gravitationally bound to the cluster, so that, if the evolutionary scheme is correct, they may still belong to the cluster after the delay needed for the onset of type 1 activity.

\subsection{Comparison with previous works on different environments: compact groups and isolated galaxies}

Compact groups. Martínez et al. (2010) found a large number of ELGs in a sample of Hickson compact groups (HCG): almost two-thirds of their sample show emission lines, and two-third of ELGs are AGNs + TOs. These values can be compared to the completeness-corrected frequencies in WINGS: $31 \%$ ELGs, of which about one-third are AGN + TO. The WINGS data therefore confirm that the AGN fraction is significantly lower in clusters than in galaxy groups with fractions doubling from clusters to groups. This result apparently holds both if AGNs are X-ray selected and if optical DDs are used. The AGN fraction in HCG galaxies with $L_{\mathrm{X}, 0.5-8.0 \mathrm{keV}} \geq 10^{41} \mathrm{erg} \mathrm{s}^{-1}$ is $0.08_{-0.01}^{+0.35}$, higher than the $\approx 5 \%$ fraction in galaxy clusters (Arnold et al. 2009; Tzanavaris et al. 2014). The value of $R_{\mathrm{N}} \gtrsim 1$ is higher for compact groups than for WINGS cluster galaxies (Martínez et al. 2010; Sohn et al. 2013; Bitsakis et al. 2015). Martínez et al. (2010) suggest that the level of activity in HCGs is characterized by a "severe deficiency of gas", and indeed the $L(\mathrm{H} \alpha)$ luminosity is comparable to that found in our cluster sample. Apparently, compact groups share some of the deficits found for clusters, although at a less extreme level. In this context, it is interesting to note that many compact group galaxies contain molecular gas that is not forming stars efficiently (Alatalo et al. 2015) and that evidence of shocks has been found in the emission line ratios of late-type galaxies in compact groups (Bitsakis et al. 2016).

The absence of type 1 AGNs has been noted in the dense environments of dynamically old compact groups (Coziol et al. 2000; Martínez et al. 2008; Bitsakis et al. 2015). In these dense environments, accretion processes in the nuclei of galaxies may be significantly affected by a hot intergalactic medium revealed through its X-ray emission. Absence of type 1 sources in dense environments has led to the suggestion that unification schemes may be dependent on active nucleus luminosities and/or environments (e.g., Dultzin-Hacyan et al. 1999, 2003; Krongold et al. 2003) or, at least, some LLAGNs may not be real AGNs.

Isolated galaxies. Isolated galaxies as defined by Verdes-Montenegro et al. (2005) represent in many ways the opposite environment from cluster galaxies, in terms of galaxy surface density: no companion galaxy of diameter $d$ within $1 / 4$ and 4 diameter of the primary should lie within $20 d$ from the primary. Isolated galaxies are believed to be sources whose properties are due to internal secular evolution and are linked to formative evolution. External influences are expected to be minimized, since isolated galaxies may not have interacted with a neighbor of significant mass in the past $\approx 3$ Gyr. Most recent studies detect emission lines in almost all galaxies (Varela et al. 2004; Hernández-Ibarra et al. 2013). The fraction of AGN depends on morphological type and luminosity, which is higher for earlier morphological types and at high luminosity (Sabater et al. 2012; Hernández-Ibarra et al. 2013), and is about $30 \%$ (without TOs) $-40 \%$ (with TOs). An even higher percentage $(\approx 2 / 3$ of sample) of AGNs + TOs was found from the analysis of an SDSS-based sample of $\sim 200$ galaxies (Coziol et al. 2011). In the luminosity domain of WINGS ELGs, $10^{39}-10^{41} \mathrm{erg} \mathrm{s}^{-1}$, AGNs and TOs are found at a percentage slightly lower than that of HII, with $R_{\mathrm{N}} \approx 0.7$ (Varela et al. 2004; Sabater et al. 2012; Hernández-Ibarra et al. 2013). The $L(\mathrm{H} \alpha)$ distributions for the four ELG classes for the isolated galaxy sample of Sabater et al. (2012, classification and line fluxes are retrieved from the Vizier catalog J/A+A/545/A15 associated 

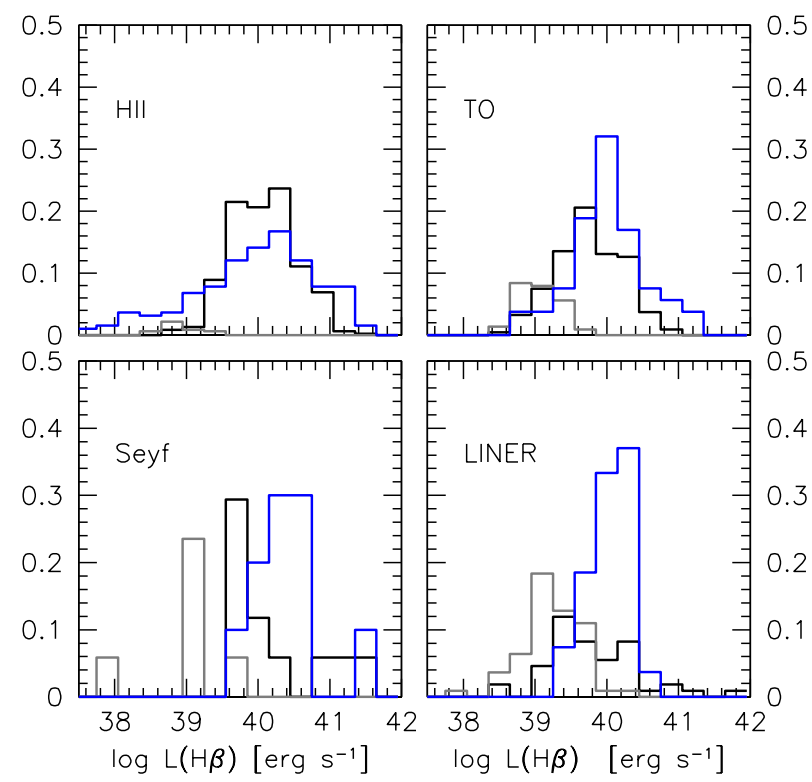

Fig. 14. Distribution of $L(\mathrm{H} \alpha)$ for the WINGS sources (w1 sample), for HII, TOs, Seyferts, and LINERs (black line), and for the sources analyzed by Sabater et al. (2012, blue). Gray lines trace the distribution of upper limits for the w0 sample. Objects classified with censored emission line ratios are included in the histogram.

with the paper) and for WINGS show that WINGS TOs and LINERs are systematically less luminous (Fig. 14).

In summary, a comparison of data related to different environments is not easy because of sample differences in luminosity and morphology. The tentative analysis of this paper indicates that there is a sequence of decreasing frequency and luminosity of ELGs from isolated galaxies to group and cluster environments, holding in the absolute magnitude domain $-23 \lesssim$ $M_{V} \lesssim-18$. The low luminosity of the emission lines makes a mechanism such as shock appealing to explain, at least in part, TOs and retired ELGs in the cluster environment. The three type 1 AGNs identified in the WINGS clusters are at clustercentric distances $\lesssim 0.5$ of the virial radius, where a significant effect from ICM is expected. As it is not possible to draw general inferences from few sources, type 1 detections in the inner regions of the WINGS clusters only suggest the need for more focused studies to analyze how hot intergalactic medium may influence nuclear accretion processes.

\section{Conclusion}

This paper presents the emission line classification for most galaxies of the WINGS - SPE survey involving X-ray luminous clusters in the redshift range 0.04-0.07. Among the immediate results of the analysis are the emission line intensities, equivalent width, estimates of fluxes, and assignment of a class on the basis of diagnostic diagrams. Sample analyses included censored data with a rigorous treatment when a two-sample comparison was carried out, and with an heuristic approach in the assignment of classification probabilities in the 2D diagnostic diagrams. The present analysis relies on ad hoc control samples that allowed us to test systematic differences between cluster and field galaxies in well-defined parameters such as $R_{\mathrm{Em}}, R_{\mathrm{N}}$, line equivalent width, and luminosities. Field galaxies were used to build control samples with statistically undistinguishable morphology mixes, luminosities, and ratio aperture-to- $R_{\mathrm{e}}$ distributions and, therefore, cannot be considered representative of a field galaxy population at low surface density.

The new analysis adds to the view of galaxies in clusters several results as follows:

1. A confirmation of the long-held notion that ELGs are less frequent in the cluster environment.

2. Detectable line emission is not only rarer, but also weaker, implying a lower amount of ionized gas per unit mass, and a lower star formation rate if the source is classified as an HII region.

3. The presence of a sizable population of sources showing spectra of TOs and LINERs. TOs and LINERs are more frequent than, or at least as frequent as in the CSs with respect to the HII sources, although they show a much lower $W(\mathrm{H} \alpha)$ than in the CSs, by a factor $\gtrsim 2-3$. The effect is illustrated well in Fig. 11.

A number of possible mechanisms can explain the LL TOs and LINERs: true low-luminosity nuclear activity, but also ionization by evolved PAGB stars and shocks. Shocks associated with the interaction between the galaxy atomic gas and the ICM provide emission line ratios in agreement with the observed ones. The phenomenon may be relatively long lived if the molecular gas in the disk of galaxies can act as a reservoir.

The relation between LL activity and cluster substructure (Ramella et al. 2007) and other properties of individual clusters (such as the entropy profile) will be investigated in an eventual paper. Such an investigation will benefit from a wider coverage of the outer cluster regions that is being provided by the ongoing extension of WINGS: OMEGAWINGS (Gullieuszik et al. 2015).

Acknowledgements. P.M. acknowledges the kind hospitality of the IAA-CSIC at Granada where an early part of this work was carried out, along with the Junta de Andalucía, through grant TIC- 114 and the Excellence Project P08-TIC-3531, and the Spanish Ministry for Science and Innovation through grants AYA201015169 for supporting her sabbatical stay in Granada.

\section{References}

Alatalo, K., Appleton, P. N., Lisenfeld, U., et al. 2015, ApJ, 812, 117 Allen, M. G., Groves, B. A., Dopita, M. A., Sutherland, R. S., \& Kewley, L. J. 2008, ApJS, 178, 20

Anderson, S. F., Margon, B., Voges, W., et al. 2007, AJ, 133, 313

Antonucci, R. 1993, ARA\&A, 31, 473

Appleton, P. N., Xu, K. C., Reach, W., et al. 2006, ApJ, 639, L51

Arnold, T. J., Martini, P., Mulchaey, J. S., Berti, A., \& Jeltema, T. E. 2009, ApJ, 707,1691

Bahcall, N. A. 1979, ApJ, 232, L83

Bahcall, N. A., \& Cen, R. 1992, ApJ, 398, L81

Balick, B., \& Heckman, T. M. 1982, ARA\&A, 20, 431

Bernardi, M., Nichol, R. C., Sheth, R. K., Miller, C. J., \& Brinkmann, J. 2006, AJ, 131, 1288

Bitsakis, T., Dultzin, D., Ciesla, L., et al. 2015, MNRAS, 450, 3114

Bitsakis, T., Dultzin, D., Ciesla, L., et al. 2016, MNRAS, 459, 957

Biviano, A., Girardi, M., Giuricin, G., Mardirossian, F., \& Mezzetti, M. 1993, ApJ, 411, L13

Biviano, A., Katgert, P., Mazure, A., et al. 1997, A\&A, 321, 84

Blanton, M. R., \& Moustakas, J. 2009, ARA\&A, 47, 159

Böhringer, H., Chon, G., \& Collins, C. A. 2014, A\&A, 570, A31

Boselli, A., \& Gavazzi, G. 2006, PASP, 118, 517

Boselli, A., Cortese, L., Boquien, M., et al. 2014, A\&A, 564, A67

Bruzual, G., \& Charlot, S. 2003, MNRAS, 344, 1000

Carrillo, R., Masegosa, J., Dultzin-Hacyan, D., \& Ordoñez, R. 1999, Rev. Mex. Astron. Astrofis., 35, 187

Cava, A., Bettoni, D., Poggianti, B. M., et al. 2009, A\&A, 495, 707

Cayatte, V., van Gorkom, J. H., Balkowski, C., \& Kotanyi, C. 1990, AJ, 100, 604

Choi, Y.-Y., Woo, J.-H., \& Park, C. 2009, ApJ, 699, 1679 
Cid Fernandes, R., Stasińska, G., Schlickmann, M. S., et al. 2010, MNRAS, 403, 1036

Contini, M., \& Aldrovandi, S. M. V. 1983, A\&A, 127, 15

Cowie, L. L., \& McKee, C. F. 1977, ApJ, 211, 135

Coziol, R., Iovino, A., \& de Carvalho, R. R. 2000, AJ, 120, 47

Coziol, R., Torres-Papaqui, J. P., Plauchu-Frayn, I., et al. 2011, Rev. Mex. Astron. Astrofis., 47, 361

Coziol, R., Torres-Papaqui, J. P., Plauchu-Frayn, I., et al. 2014, Rev. Mex. Astron. Astrofis., 50, 255

Dale, D. A., Giovanelli, R., Haynes, M. P., Hardy, E., \& Campusano, L. E. 2001, AJ, 121, 1886

De Grandi, S., Guzzo, L., Böhringer, H., et al. 1999, ApJ, 513, L17

D’Onofrio, M., Bindoni, D., Fasano, G., et al. 2014, A\&A, 572, A87

D’Onofrio, M., Marziani, P., \& Buson, L. 2015, Frontiers in Astronomy and Space Sciences, 2, 4

Dressler, A., Thompson, I. B., \& Shectman, S. A. 1985, ApJ, 288, 481

Dultzin-Hacyan, D., Krongold, Y., Fuentes-Guridi, I., \& Marziani, P. 1999, ApJ, 513, L111

Dultzin-Hacyan, D., Krongold, Y., \& Marziani, P. 2003, in Rev. Mex. Astron. Astrofis. Conf. Ser. 17, eds. V. Avila-Reese, C. Firmani, C. S. Frenk, \& C. Allen, 79

Ehlert, S., von der Linden, A., Allen, S. W., et al. 2014, MNRAS, 437, 1942

Fang, J. J., Faber, S. M., Salim, S., Graves, G. J., \& Rich, R. M. 2012, ApJ, 761 23

Fasano, G., Marmo, C., Varela, J., et al. 2006, A\&A, 445, 805

Fasano, G., Marmo, C., Varela, J., et al. 2012, MNRAS, 420, 926

Feigelson, E. D., \& Nelson, P. I. 1985, ApJ, 293, 192

Ferland, G. J., \& Netzer, H. 1983, ApJ, 264, 105

Fritz, J., Poggianti, B. M., Cava, A., et al. 2011, A\&A, 526, A45

Fritz, J., Poggianti, B. M., Cava, A., et al. 2014, A\&A, 566, A32

Giovanelli, R., \& Haynes, M. P. 1985, ApJ, 292, 404

Girardi, M., Giuricin, G., Mardirossian, F., Mezzetti, M., \& Boschin, W. 1998, ApJ, 505, 74

Gisler, G. R. 1978, MNRAS, 183, 633

González-Martín, O., Masegosa, J., Márquez, I., Guerrero, M. A., \& Dultzin-Hacyan, D. 2006, A\&A, 460, 45

González-Martín, O., Masegosa, J., Márquez, I., et al. 2015, A\&A, 578, A74

Gullieuszik, M., Poggianti, B., Fasano, G., et al. 2015, A\&A, 581, A41

Gunn, J. E., \& Gott, III, J. R. 1972, ApJ, 176, 1

Haggard, D., Green, P. J., Anderson, S. F., et al. 2010, ApJ, 723, 1447

Halpern, J. P., \& Steiner, J. E. 1983, ApJ, 269, L37

Hao, L., Strauss, M. A., Tremonti, C. A., et al. 2005, AJ, 129, 1783

Heckman, T. M. 1980, A\&A, 87, 152

Heckman, T. M., \& Best, P. N. 2014, ARA\&A, 52, 589

Hernández-Ibarra, F. J., Dultzin, D., Krongold, Y., et al. 2013, MNRAS, 434, 336

Hidaka, M., \& Sofue, Y. 2002, PASJ, 54, 223

Ho, L. C. 2005, Ap\&SS, 300, 219

Ho, L. C., Filippenko, A. V., \& Sargent, W. L. 1995, ApJS, 98, 477

Ho, L. C., Filippenko, A. V., Sargent, W. L. W., \& Peng, C. Y. 1997, ApJS, 112, 391

Hopkins, A. M., Miller, C. J., Nichol, R. C., et al. 2003, ApJ, 599, 971

Huchra, J., \& Burg, R. 1992, ApJ, 393, 90

Hwang, H. S., Park, C., Elbaz, D., \& Choi, Y.-Y. 2012, A\&A, 538, A15

Kauffmann, G. 2009, A\&A, 500, 201

Kauffmann, G., Heckman, T. M., Tremonti, C., et al. 2003, MNRAS, 346, 1055

Kauffmann, G., White, S. D. M., Heckman, T. M., et al. 2004, MNRAS, 353, 713

Kennicutt, Jr., R. C. 1998, ARA\&A, 36, 189

Kewley, L. J., Dopita, M. A., Sutherland, R. S., Heisler, C. A., \& Trevena, J. 2001, ApJ, 556, 121

Kewley, L. J., Groves, B., Kauffmann, G., \& Heckman, T. 2006, MNRAS, 372, 961

Koulouridis, E., \& Plionis, M. 2010, ApJ, 714, L181

Koulouridis, E., Plionis, M., Chavushyan, V., 2013, A\&A, 552, A135

Kriss, G. 1994, Astronomical Data Analysis Software and Systems III, ASP Conf. Ser., 61, 437

Krolik, J. H. 1999, Active galactic nuclei: from the central black hole to the galactic environment (Princeton University Press)

Krongold, Y., Dultzin-Hacyan, D., \& Marziani, P. 2003, in Active Galactic Nuclei: From Central Engine to Host Galaxy, eds. S. Collin, F. Combes, \& I. Shlosman, ASP Conf. Ser., 290, 523
Larson, R. B., \& Tinsley, B. M. 1978, ApJ, 219, 46

Lee, H., McCall, M. L., \& Richer, M. G. 2003, AJ, 125, 2975

Madau, P., \& Dickinson, M. 2014, ARA\&A, 52, 415

Madau, P., Pozzetti, L., \& Dickinson, M. 1998, ApJ, 498, 106

Manzer, L. H., \& De Robertis, M. M. 2014, ApJ, 788, 140

Márquez, I., González-Martín, O., Masegosa, J., Guerrero, M. A., \& Dultzin-Hacyan, D. 2007, in The Central Engine of Active Galactic Nuclei, eds. L. C. Ho, \& J.-W. Wang, ASP Conf. Ser., 373, 542

Martínez, M. A., del Olmo, A., Coziol, R., \& Focardi, P. 2008, ApJ, 678, L9

Martínez, M., Del Olmo, A., Coziol, R., Perea, J., \& Focardi, P. 2010, in Galaxies in Isolation: Exploring Nature Versus Nurture, eds. L. Verdes-Montenegro, A. Del Olmo, \& J. Sulentic, ASP Conf. Ser., 421, 125

Martini, P., Kelson, D. D., Kim, E., Mulchaey, J. S., \& Athey, A. A. 2006, ApJ, 644, 116

Martini, P., Sivakoff, G. R., \& Mulchaey, J. S. 2009, ApJ, 701, 66

Marziani, P., Keel, W. C., Dultzin-Hacyan, D., \& Sulentic, J. W. 1994, ApJ, 435, 668

Marziani, P., Dultzin-Hacyan, D., Krongold, Y., \& D’Onofrio, M. 2001, in The Central Kiloparsec of Starbursts and AGN: The La Palma Connection, eds. J. H. Knapen, J. E. Beckman, I. Shlosman, \& T. J. Mahoney, ASP Conf. Ser., 249, 284

Marziani, P., D’Onofrio, M., Bettoni, D., et al. 2013, Astron. Nachr., 334, 412

Merluzzi, P., Busarello, G., Dopita, M. A., et al. 2013, MNRAS, 429, 1747

Miller, C. J., Nichol, R. C., Gómez, P. L., Hopkins, A. M., \& Bernardi, M. 2003, ApJ, 597, 142

Monreal-Ibero, A., Arribas, S., Colina, L., et al. 2010, A\&A, 517, A28

Moore, B., Katz, N., Lake, G., Dressler, A., \& Oemler, A. 1996, Nature, 379, 613

Moretti, A., Poggianti, B. M., Fasano, G., et al. 2014, A\&A, 564, A138

Newman, S. F., Buschkamp, P., Genzel, R., et al. 2014, ApJ, 781, 21

Ogle, P., Antonucci, R., Appleton, P. N., \& Whysong, D. 2007, ApJ, 668, 699

Osterbrock, D. E., \& Ferland, G. J. 2006, in Astrophysics of gaseous nebulae and active galactic nuclei (University Science Books)

Padovani, P., \& Matteucci, F. 1993, ApJ, 416, 26

Park, C., \& Hwang, H. S. 2009, ApJ, 699, 1595

Pimbblet, K. A., Shabala, S. S., Haines, C. P., Fraser-McKelvie, A., \& Floyd, D. J. E. 2013, MNRAS, 429, 1827

Poggianti, B. M. 1997, A\&AS, 122, 399

Poggianti, B. M., von der Linden, A., De Lucia, G., et al. 2006, ApJ, 642, 188

Poggianti, B. M., von der Linden, A., De Lucia, G., et al. 2016, AJ, 151, 78

Ramella, M., Biviano, A., Pisani, A., et al. 2007, A\&A, 470, 39

Reiprich, T. H., \& Böhringer, H. 2002, ApJ, 567, 716

Rich, J. A., Kewley, L. J., \& Dopita, M. A. 2011, ApJ, 734, 87

Rola, C., \& Pelat, D. 1994, A\&A, 287, 676

Sabater, J., Verdes-Montenegro, L., Leon, S., Best, P., \& Sulentic, J. 2012, A\&A, 545, A 15

Sabater, J., Best, P. N., \& Argudo-Fernández, M. 2013, MNRAS, 430, 638

Scoville, N., Arnouts, S., Aussel, H., et al. 2013, ApJS, 206, 3

Sivanandam, S., Rieke, M. J., \& Rieke, G. H. 2010, ApJ, 717, 147

Sivanandam, S., Rieke, M. J., \& Rieke, G. H. 2014, ApJ, 796, 89

Sohn, J., Hwang, H. S., Lee, M. G., Lee, G.-H., \& Lee, J. C. 2013, ApJ, 771, 106

Spitzer, Jr., L., \& Baade, W. 1951, ApJ, 113, 413

Stasińska, G., Vale Asari, N., Cid Fernandes, R., et al. 2008, MNRAS, 391, L29

Stasińska, G., Costa-Duarte, M. V., Vale Asari, N., Cid Fernandes, R., \& Sodré, L. 2015, MNRAS, 449, 559

Sulentic, J. W. 1976, ApJS, 32, 171

Tzanavaris, P., Gallagher, S. C., Hornschemeier, A. E., et al. 2014, ApJS, 212, 9

Valentinuzzi, T., Poggianti, B. M., Fasano, G., et al. 2011, A\&A, 536, A34

Varela, J., Moles, M., Márquez, I., et al. 2004, A\&A, 420, 873

Varela, J., D'Onofrio, M., Marmo, C., et al. 2009, A\&A, 497, 667

Veilleux, S., \& Osterbrock, D. E. 1987, ApJS, 63, 295

Verdes-Montenegro, L., Sulentic, J., Lisenfeld, U., et al. 2005, A\&A, 436, 443

Veron, P., Goncalves, A. C., \& Veron-Cetty, M.-P. 1997, A\&A, 319, 52

Viegas-Aldrovandi, S. M., \& Gruenwald, R. B. 1990, ApJ, 360, 474

Villarroel, B., \& Korn, A. J. 2014, Nat. Phys., 10, 417

von der Linden, A., Wild, V., Kauffmann, G., White, S. D. M., \& Weinmann, S. 2010, MNRAS, 404, 1231 


\section{Appendix A: Error analysis and treatment of censored data}

Errors on the logarithm of line intensity ratios are in general symmetric since errors on intensity ratios follow lognormal distributions. In the present paper, errors were estimated following the prescription of Rola \& Pelat (1994), which assumes a proper lognormal distribution for the case of modest values of $I_{\mathrm{p}} / \mathrm{rms}$ $\left(I_{\mathrm{p}} / \mathrm{rms} \lesssim 10\right.$, as is the case of most of our measurements). Errors on logarithm of line intensity ratios are significantly asymmetric in the case in which the two lines have different $I_{\mathrm{p}} / \mathrm{rms}$, and one of them has $I_{\mathrm{p}} / \mathrm{rms} \lesssim 10$.

Upper and lower limits were included in the analysis. Emission lines whose $I_{\mathrm{p}} / \mathrm{rms}$ is below the limiting values (either 3 or 4) were considered not detected, and an upper limit was set right at the limiting $I_{\mathrm{p}} / \mathrm{rms}$ value. Both upper and lower limits in line ratios should be included in the intensity ratio and diagnostic analysis to fully take advantage of the generality of the selection criteria of Expression 2 since $\mathrm{H} \beta$ and $\mathrm{H} \alpha$ intensity appear at the denominator.

Considering only detections would be equivalent to setting an ill-defined limit to the $\mathrm{H} \beta$ equivalent width. The broad range of $\mathrm{S} / \mathrm{N}$ present in the WINGS spectra introduced a censoring on emission line detection that is dependent on the line equivalent width. Measuring the equivalent width on mock spectra built with (1) the stellar template of a quiescent population (i.e., the most likely case); (2) an artificial unresolved emission line meant to mimic $\mathrm{H} \beta$; and (3) Gaussian noise, show that the minimum equivalent width as a function of $\mathrm{S} / \mathrm{N}$ is given by

$W_{\min } \approx 18.2\left(\frac{I_{\mathrm{p}}}{\mathrm{rms}}\right)_{\min , 3}\left(\frac{\mathrm{S}}{\mathrm{N}}\right)^{-1.10} \AA$

where the relation is given for $\left(\frac{I_{\mathrm{p}}}{\mathrm{rms}}\right)_{\min }=3$. The signal-to-noise ratio was measured in correspondence of [OIII] $\lambda 5007$, and is meant to be representative of the overall $\mathrm{S} / \mathrm{N}$ of each spectrum. A $S / N \approx 6$ would correspond to a minimum $W_{\min } \approx 3 \AA$. This equivalent width limit was applied in our preliminary analysis (Marziani et al. 2013); however from the distribution of $\mathrm{S} / \mathrm{N}$ versus $W_{\min }$ we deduce that a large number of sources with detected emission in higher $\mathrm{S} / \mathrm{N}$ spectra were not considered (Fig. A.1).

\section{A.1. Analysis of diagnostic ratios and diagrams}

The four diagnostic diagrams based on pairs of emission line ratios and employed in this study $\left(\mathrm{DD}_{[\mathrm{NII}]}, \mathrm{DD}_{[\mathrm{OII}]}, \mathrm{DD}_{[\mathrm{OI}]}\right.$, $\left.\mathrm{DD}_{[\mathrm{SII}]}\right)$ are not equivalent. Each of these DDs provide different information on the physical conditions of the emission line gas. In addition, the interpretation is affected by instrumental problems specific to each diagram: the $\mathrm{DD}_{[\mathrm{OII}]}$ is efficient in detecting ELGs since it involves the strong [OII] $\lambda 3727$ doublet. However, the use of this line for diagnostic analysis necessitates a reliable correction for internal extinction. For the $\approx 1300$ sources that lack coverage of $\mathrm{H} \alpha$ (northern sample), this correction cannot be computed. The $\mathrm{DD}_{[\mathrm{NII}]}$ is by far, from the point of view of measurement robustness, the most valuable diagram: it is not strongly affected by internal extinction and involves the strongest lines observed in the spectral range of WINGS - SPE. It also allows for the finest classification, separating objects into four classes (HII, transition objects, LINERs, and Seyferts; Kewley et al. 2001, 2006, Sect. 4.5). Low ionization lines like [OI] $\lambda 6300$ and [SII] $\lambda \lambda 6716,6731$ are enhanced in an extended post-shock recombination region (analogous to

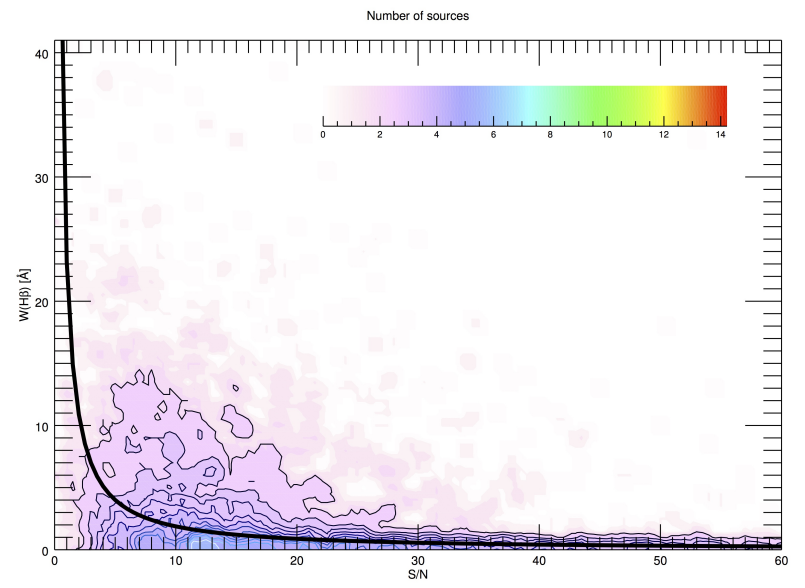

Fig. A.1. Minimum $W(\mathrm{H} \beta)$ (thick line) and the distribution of $W(\mathrm{H} \beta)$ in the plane $W(\mathrm{H} \beta)$ vs. $\mathrm{S} / \mathrm{N}$. Isoplets represent source numbers for the sample obtained joining w0 and w1, color-coded as shown by the bar at the top right corner of the plot.

the partially-ionized zone created by the AGN soft X-ray radiation; Krolik 1999, and references therein). In a low-luminosity context their detection may indicate well either shock or AGN photoionization as a production mechanism. They are especially useful to test the nature of TOs (Veron et al. 1997). However, the [OI] $\lambda 6300$ and [SII] $\lambda \lambda 6716,6731$ lines, even if enhanced, remain rather weak, and are often of uncertain detection with WINGS - SPE data. Their use for individual sources should be therefore strictly restricted to detections.

\section{A.1.1. Assigning the probability of classification}

A quantitative analysis of the diagnostic diagrams requires (1) a proper consideration of the source position in the DD with respect to the limits drawn to distinguish different ELG classes; (2) an adequate treatment of uncertainties and upper limits, also aimed at providing a probability that a source is properly classified. This approach is especially needed since (1) there is a large clustering of sources close to the dividing lines, for example, at the boundary between Seyferts and HII regions; and (2) TOs are located in a narrow strip between HII, Seyferts, and LINERs.

We considered the probability $P_{i, j}$ that a source in region $i$ could be classified as belonging to region $j$ in a diagnostic diagram involving ratios $r_{1}$ and $r_{2}$. The case $j=i$ corresponds to a correct classification in the region of the diagram where the source is actually located, and the indexes $i$ and $j$ take the four values HII, LINERs, TO, and Seyferts. In other words, $P_{i, i}$ with $i=1$ is the probability that a source classified as HII, is really HII, $P_{i, j}$ with $i=2$, and $j=1$ is the probability that a source falling in the TO domain is misclassified, and that the correct classification is HII. Clearly, $\sum_{j} P_{i, j}=1, \forall i . P_{i, j}$ can be written as follows:

$$
\begin{aligned}
P_{i, j}= & \int_{r_{1, j \min }}^{r_{1, j, \max }}\left(\int_{r_{2, j, \min }\left(r_{1}\right)}^{r_{2, j \max }\left(r_{1}\right)} \pi_{2}\left(r_{2}\right) \mathrm{d} r_{2}\right) \pi_{1}\left(r_{1}\right) \mathrm{d} r_{1} \\
P_{i, j}= & \int_{r_{1, j, \min }}^{r_{1, j, \max }}\left\{\Pi_{2}\left(r_{2, j, \max }\left(r_{1}\right)\right)-\Pi_{2}\left(r_{2, j, \min }\left(r_{1}\right)\right)\right\} \\
& \pi_{1}\left(r_{1}\right) \mathrm{d} r_{1} .
\end{aligned}
$$

We described here a DD as a plane with a dependence between the ratio on ordinate $r_{2}$ on the ratio $r_{1}$ on abscissa, $r_{2, j, \max }\left(r_{1}\right)$ 
and $r_{2, j \min }\left(r_{1}\right)$. The probabilities are assigned assuming that errors on ratios follow a lognormal distribution in the case of detection and define a probability density $\pi$ for each diagnostic ratio. The value $\Pi_{2}$ is the cumulative distribution integrated over $r_{2}$ that is a function of $r_{1}$ since the limits on $r_{2}$ are in general a function of $r_{1}$ given by dividing lines of Kewley et al. (2001) and Kauffmann et al. (2003). An underlying assumption is that the joint probability density $\pi$ can be factored as $\pi=\pi_{1}\left(r_{1}\right) \cdot \pi_{2}\left(r_{2}\right)$, i.e., that the probabilities of the two diagnostic ratios are independent. A similar approach was followed by Manzer \& De Robertis (2014).

The probability can also be assigned in case of upper and lower limits, considering the Kaplan-Meier (KM) estimator of the cumulative survival function (Feigelson \& Nelson 1985). To keep our approach simple we approximate these cumulative distributions with an error function and the probability density with Gaussian distributions in the log-log plane for the diagnostic ratios [OIII] $\lambda 5007 / \mathrm{H} \beta,[\mathrm{NII}] \lambda 6583 / \mathrm{H} \alpha$, and $[\mathrm{OII}] \lambda 3727 / \mathrm{H} \beta$, along with the probability density field associated with each KM estimator. The probability density is needed to compute the integral of Eq. (A.2) in case a censored value appears on the ratio in abscissa. If this approach is followed, it is possible to define a $P_{\mathrm{ij}}$ for $i=$ Seyfert, HII, TO, and LINER. Censored data analysis is rigorous as long as line equivalent width and single intensity ratios are considered, but there is no established solution to the statistical problem of univariate or 2D censoring with mixed censoring (i.e., upper and lower limits). The KM estimators were computed considering detections $+\mathrm{UL}$ and detections + LL separately. The value of the probability estimates, if upper (occurring most often) and lower limits (rarer) are included, will be therefore heuristic. In addition, the K-M estimator of the survival function depends on the observed distribution of data points, which in turns depends on the instrumental capabilities of the survey, as well as on the intrinsic physical properties of the sample that is observed. Nonetheless, consideration of censored data provides a more realistic view of important aspects related to the prevalence of ELG classes, and to the difference in distribution of parameters such as line luminosity and equivalent width. Probabilities including upper limits were assigned and are reported in the database table (Table B.1, P_OII_..., P_NII_..., etc. keys). 


\section{Appendix B: Additional table}

Table B.1. Description of fields in the emission line catalog of the WINGS database.

\begin{tabular}{|c|c|c|c|c|}
\hline $\mathrm{COL}$ & Identifier & Type & Units & Description \\
\hline 1 & WID & CHAR & NULL & WINGS identifier \\
\hline 2 & CLU & CHAR & NULL & Cluster identification code \\
\hline 3 & MEM & INTEGER & NULL & Membership class: 1 member of cluster, 0 : non-member \\
\hline 4 & IDS & CHAR & NULL & File name with spectrum aperture number \\
\hline 5 & SN & FLOAT & NULL & $1 \sigma \mathrm{S} / \mathrm{N}$ measured in correspondence of the $[\mathrm{OIII}] \lambda 5007$ line \\
\hline 6 & R_OII & FLOAT & NULL & Ratio [OII] $\lambda 3727 / \mathrm{rms}_{[\mathrm{OII}]}$ \\
\hline 7 & R_HB & FLOAT & NULL & Ratio $\mathrm{H} \beta / \mathrm{rms}_{\mathrm{H} \beta}$ \\
\hline 8 & R_OIII & FLOAT & NULL & Ratio [OIII] $\lambda 5007 / \mathrm{rms}_{[\mathrm{OIII}]}$ \\
\hline 9 & R_OI & FLOAT & NULL & Ratio [OI] $\lambda 6300 / \mathrm{rms}_{[}[\mathrm{OI}]$ \\
\hline 10 & R_HA & FLOAT & NULL & Ratio $\mathrm{H} \alpha / \mathrm{rms}_{\mathrm{H} \alpha}$ \\
\hline 11 & R_NII & FLOAT & NULL & Ratio $[\mathrm{NII}] \lambda 6583 / \mathrm{rms}_{[\mathrm{NII}]}$ \\
\hline 12 & R_SII & FLOAT & NULL & Ratio [SII] $] \lambda \lambda 6716,6731 / \mathrm{rms}_{[\mathrm{SII}]}$ \\
\hline 13 & DETECT & CHAR & NULL & Detection of emission lines following criterion of Eq. (2). \\
\hline 14 & EW_MIN & FLOAT & $\AA$ & Minimum equation width $W$ detectable at $\mathrm{S} / \mathrm{N}$ reported in Col. 4 , from Eq. (A.1) \\
\hline 15 & EW_HB & FLOAT & $\AA$ & Rest-frame equivalent width of $\mathrm{H} \beta$ \\
\hline 16 & EW_HB_ERR & FLOAT & $\AA$ & Rest-frame $\mathrm{H} \beta$ equivalent width error \\
\hline 17 & EW_HB_CENSOR & INTEGER & NULL & Rest-frame $\mathrm{H} \beta$ equivalent censorship \\
\hline 18 & EW_HA & FLOAT & $\AA$ & Rest-frame equivalent width of $\mathrm{H} \alpha$ \\
\hline 19 & EW_HA_ERR & FLOAT & $\AA$ & Rest-frame $\mathrm{H} \alpha$ equivalent width error \\
\hline 20 & EW_HA_CENSOR & INTEGER & NULL & Rest-frame $\mathrm{H} \alpha$ equivalent censorship \\
\hline 21 & R_OIIHB & FLOAT & NULL & Decimal logarithm of ratio [OII] $\lambda 3727 / \mathrm{H} \beta$ \\
\hline 22 & R_OIIHB_ERRM & FLOAT & NULL & Lower error on $\log [\mathrm{OII}] \lambda 3727 / \mathrm{H} \beta$ \\
\hline 23 & R_OIIHB_ERRP & FLOAT & NULL & Upper error on $\log [\mathrm{OII}] \lambda 3727 / \mathrm{H} \beta$ \\
\hline 24 & R_OIIHB_CENSOR & INTEGER & NULL & Censorship on $\log [\mathrm{OII}] \lambda 3727 / \mathrm{H} \beta$ \\
\hline 25 & R_OIIIHB & FLOAT & NULL & Decimal logarithm of ratio $[\mathrm{OIII}] \lambda 5007 / \mathrm{H} \beta$ \\
\hline 26 & R_OIIIHB_ERR & FLOAT & NULL & Lower error on $\log [\mathrm{OIII}] \lambda 5007 / \mathrm{H} \beta$ \\
\hline 27 & R_OIIIHB_ERR & FLOAT & NULL & Upper error on $\log [\mathrm{OIII}] \lambda 5007 / \mathrm{H} \beta$ \\
\hline 28 & R_OIIIHB_CENSOR & INTEGER & NULL & Censorship on $\log [\mathrm{OIII}] \lambda 5007 / \mathrm{H} \beta$ \\
\hline 29 & R_OIHA & FLOAT & NULL & Decimal logarithm of ratio $[\mathrm{OI}] \lambda 6300 / \mathrm{H} \alpha$ \\
\hline 30 & R_OIHA_ERRM & FLOAT & NULL & Lower error on $\log [\mathrm{OI}] \lambda 6300 / \mathrm{H} \alpha$ \\
\hline 31 & R_OIHA_ERRP & FLOAT & NULL & Upper error on $\log [\mathrm{OI}] \lambda 6300 / \mathrm{H} \alpha$ \\
\hline 32 & R_OIHA_CENSOR & INTEGER & NULL & Censorship on $\log [\mathrm{OI}] \lambda 6300 / \mathrm{H} \alpha$ \\
\hline 33 & R_NIIHA & FLOAT & NULL & Decimal logarithm of ratio $[\mathrm{NII}] \lambda 6583 / \mathrm{H} \alpha$ \\
\hline 34 & R_NIIHA_ERRM & FLOAT & NULL & Lower error on $\log [\mathrm{NII}] \lambda 6583 / \mathrm{H} \alpha$ \\
\hline 35 & R_NIIHA_ERRP & FLOAT & NULL & Upper error on $\log [\mathrm{NII}] \lambda 6583 / \mathrm{H} \alpha$ \\
\hline 36 & R_NIIHA_CENSOR & INTEGER & NULL & Censorship on $\log [\mathrm{NII}] \lambda 6583 / \mathrm{H} \alpha$ \\
\hline 37 & R_SIIHA & FLOAT & NULL & Decimal logarithm of ratio $[\mathrm{SII}] \lambda \lambda 6716,6731 / \mathrm{H} \alpha$ \\
\hline 38 & R_SIIHA_ERRM & FLOAT & NULL & Lower error on $\log [\mathrm{SII}] \lambda \lambda 6716,6731 / \mathrm{H} \alpha$ \\
\hline 39 & R_SIIHA_ERRP & FLOAT & NULL & Upper error on $\log [\mathrm{SII}] \lambda \lambda 6716,6731 / \mathrm{H} \alpha$ \\
\hline 40 & R_SIIHA_CENSOR & INTEGER & NULL & Censorship on $\log [\mathrm{SII}] \lambda \lambda 6716,6731 / \mathrm{H} \alpha$ \\
\hline 41 & CLAASS_ÖII & CHAR & NULL & Class from location in $[\mathrm{OII}] \lambda 3727 \mathrm{DD}$ \\
\hline 42 & P_OII_HII & FLOAT & NULL & Probability of HII classification in [OII] $\lambda 3727$ DD \\
\hline 43 & P_OII_LIN & FLOAT & NULL & Probability of LINER classification in [OII] $\lambda 3727$ DD \\
\hline 44 & P_OII_SEYF & FLOAT & NULL & Probability of Seyfert classification in [OII] $\lambda 3727$ DD \\
\hline 45 & CLASS_OII_REV & CHAR & NULL & Revised class from location in [OII] $\lambda 3727 \mathrm{DD}$ and probability \\
\hline 46 & CLASS_OI & CHAR & NULL & Class from location in $[\mathrm{OI}] \lambda 6300 \mathrm{DD}$ \\
\hline 47 & CLASS_NII & CHAR & NULL & Class from location in $\mathrm{DD}_{[\mathrm{NII}]}$ \\
\hline 48 & P_NII_HII & FLOAT & NULL & Probability of HII classification in $\mathrm{DD}_{[\mathrm{NII}]}$ \\
\hline 49 & P_NII_TO & FLOAT & NULL & Probability of TO classification in $\mathrm{DD}_{[\mathrm{NII}]}$ \\
\hline 50 & P_NII_LIN & FLOAT & NULL & Probability of LINER classification in $\mathrm{DD}_{[\mathrm{NII}]}$ \\
\hline 51 & P_NII_SEYF & FLOAT & NULL & Probability of Seyfert classification in $\mathrm{DD}_{[\mathrm{NII}]}$ \\
\hline 52 & CLASS_NII_REV & CHAR & NULL & Revised class from location in $[\mathrm{NII}] \lambda 6583 \mathrm{DD}$ and probability \\
\hline 53 & CLASS_SII & CHAR & NULL & Class from location in $[\mathrm{SII}] \lambda \lambda 6716,6731 \mathrm{DD}$ \\
\hline 54 & FL & FLOAT & $\operatorname{erg~s}^{-1} \mathrm{~cm}^{-2} \AA^{-1}$ & Specific flux at $5000 \AA$ \\
\hline 55 & FLV & FLOAT & $\operatorname{erg~s}^{-1} \mathrm{~cm}^{-2} \AA^{-1}$ & Specific flux from $V$-band \\
\hline 56 & L_HB & FLOAT & $\operatorname{erg~s}^{-1}$ & Decimal log of $\mathrm{H} \beta$ emission line luminosity \\
\hline 57 & L_HB_CENSOR & INTEGER & NULL & $\mathrm{H} \beta$ emission line luminosity censorship flag \\
\hline 58 & L_HA & FLOAT & $\mathrm{erg} \mathrm{s}^{-1}$ & Decimal $\log$ of $\mathrm{H} \alpha$ emission line luminosity \\
\hline 59 & L_HA_CENSOR & INTEGER & NULL & H $\alpha$ emission line luminosity censorship flag \\
\hline 60 & NOTES & CHAR & NULL & Comments on individual sources \\
\hline
\end{tabular}

